\title{
An Integrated Statewide Ocean Data Network: Providing Data Analysis Necessary for Integrated Multi-use Ocean Management in Massachusetts
}

July 2009

Prepared by:

Applied Science Associates, Inc. and

The Massachusetts Ocean Partnership

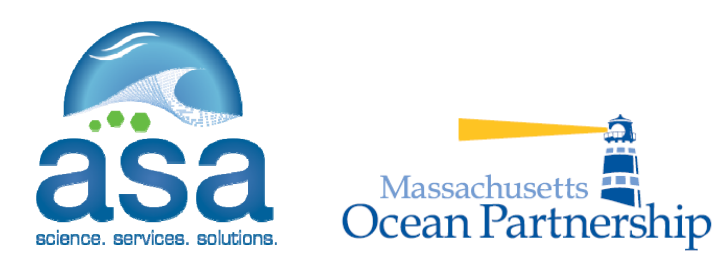





\section{Table of Contents}

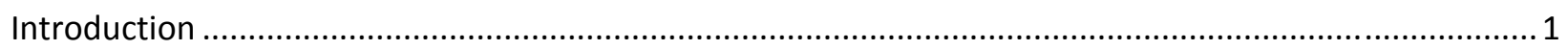

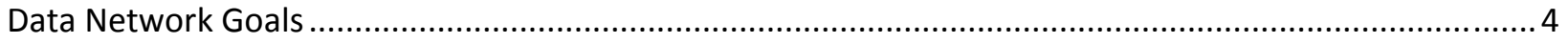

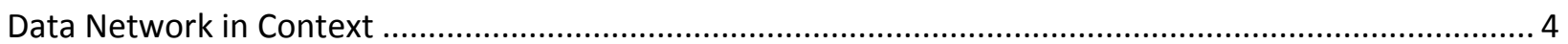

GOOS

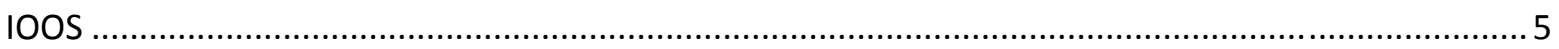

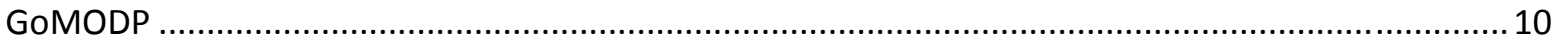

$\mathrm{OOI}$

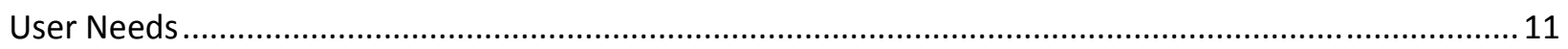

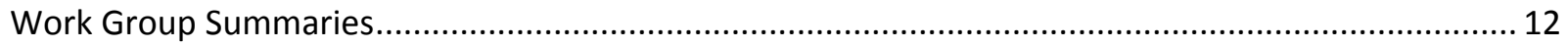

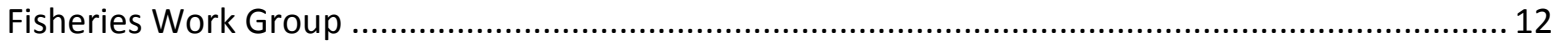

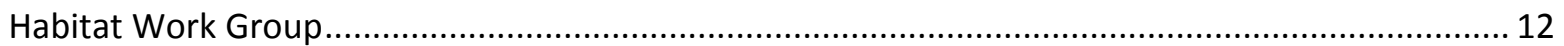

Ocean Recreational and Cultural Services Work Group ............................................................... 13

Transportation, Navigation and Infrastructure Work Group.......................................................... 13

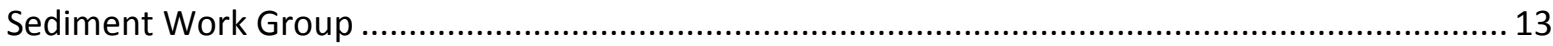

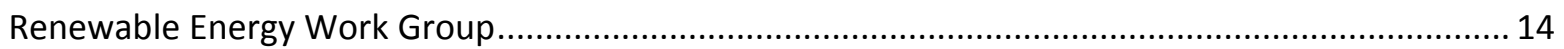

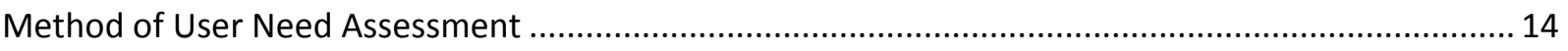

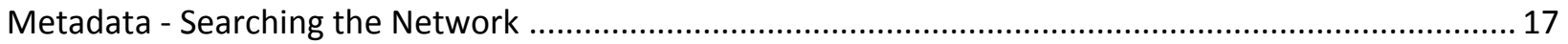

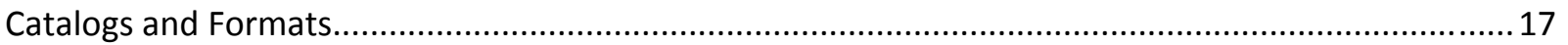

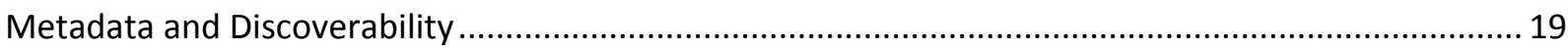

Data Categories

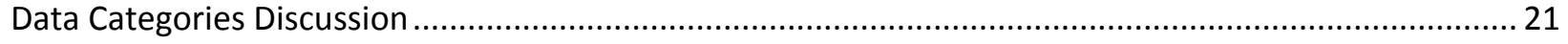

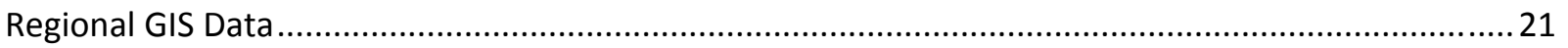

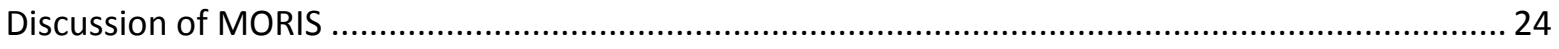

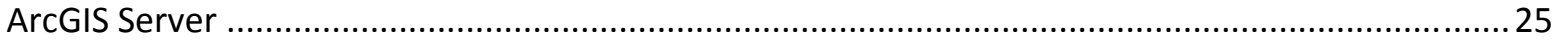

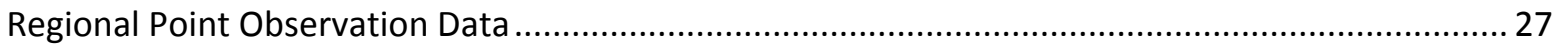

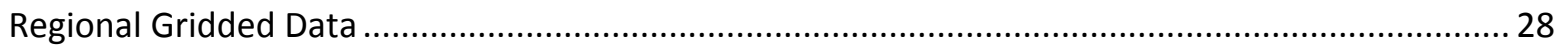

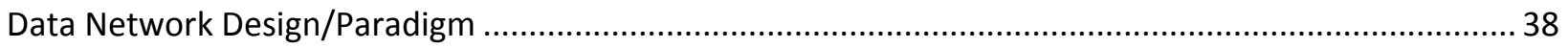

Focus on Interoperability and the use of existing science tools connected to commercial web 2.0

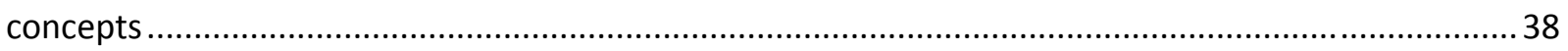

Leverage existing global, national, and regional efforts and use the general guidelines provided by the National IOOS DMAC/DIF concept of operations 
Provide tools and "glue" to integrate legacy systems as opposed to redesigning systems .................. 41

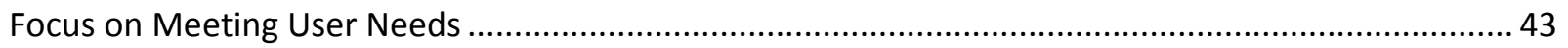

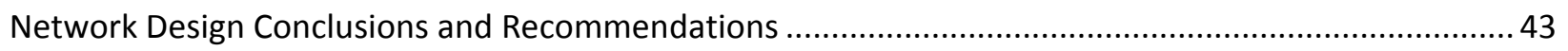

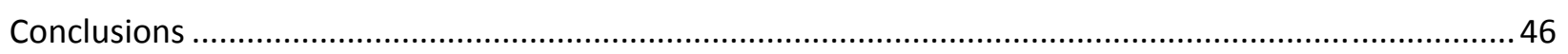

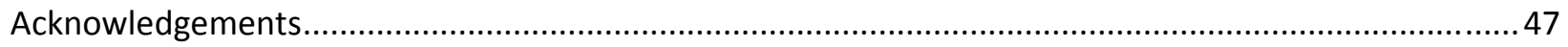

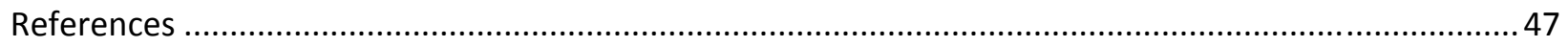

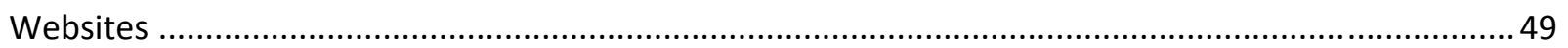

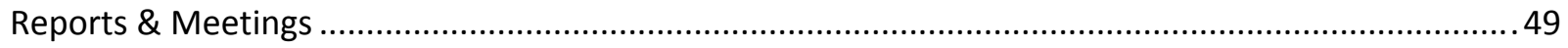

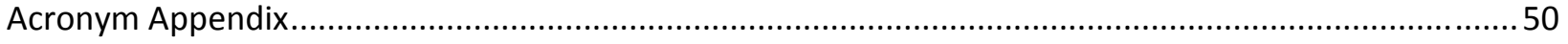




\section{Introduction}

In October 2008, the Massachusetts Ocean Partnership (MOP) contracted with Applied Science Associates (ASA) to provide consultation in support of an ocean data network to facilitate integrated multi-use ocean management in Massachusetts and support development of the Ocean Management Plan for Massachusetts. This work was contracted through its fiscal sponsor The University of Massachusetts Boston (UMB) and in coordination with the Massachusetts Executive Office of Energy and Environmental Affairs (EOEEA), the Massachusetts Office of Coastal Zone Management (CZM) and the Massachusetts Office of Geographic and Environmental Information (MassGIS).

An ocean data network is an infrastructure of data, systems, services, and tools that allow a variety of users including the public, coastal managers, and research scientists to access "live" and archived data related to coastal and ocean management. This may include maps, observations, and model data. The goal of the Massachusetts Ocean Plan is common to many state and federal agencies. A considerable amount of work has already been completed by these agencies and MOP should take advantage of existing systems while taking the lead in strategic places to connect the pieces.

We will not discuss the need for ocean observing and the economic benefits; there are numerous reports that cover this topic ranging from the need to monitor climate change, to assessing renewable energy benefits (Hauke L. Kite-Powell et al.. 2005). These reports suggest that the societal benefits of ocean observing are several hundreds of millions of dollars per year and recommend

"Cost efficient and effective means of communicating the information derived from the ocean observations to users in a timely manner," and that users should be able to "effectively incorporate the information into their decisions."

In order to meet these goals the design concepts that we will focus on are:

- Focus on interoperability and the use of existing science tools connected to commercial Web 2.0 concepts

- Leverage existing global, national, and regional efforts and use the general guidelines provided by the National IOOS DMAC concept of operations

- Provide tools and "glue" to integrate legacy systems as opposed to redesigning components

- Focus on meeting user's needs

This report reviews existing observation systems, regional initiatives, existing infrastructure, data management concepts, and user issues. There are many active, high quality systems in place, both regional and national, and groups of dedicated scientists and data managers already working on data management systems. These systems generally live in isolation from the Web 2.0 world of rapidly evolving technologies to search and share data. Our recommendation is to leverage these science systems and the data that they provide, with the latest commonly available technology.

As part of the process to evaluate existing initiatives, we performed extensive research, including several interviews with scientists and technologists from different communities. We also relied on existing research and findings from a wide variety of sources, including the following programs and technologies:

\section{- 1 - INTERIM DRAFT}


- Ocean Observatories Initiative (OOI) - National Science Foundation's contribution to the U.S. Integrated Ocean Observing System (IOOS). This initiative looks to discoveries enabled by new technologies to construct a long-term network infrastructure of science-driven sensor systems to measure the physical, chemical, geological and biological variables in the ocean and seafloor.

- Ocean Biogeographical Information System (OBIS) - an evolving strategic alliance of people and organizations sharing a vision to make marine biogeographical data, from all over the world, freely available over the World Wide Web.

- National Water Quality Monitoring Network (NWQMN) - an interagency network providing information based on common criteria and standards about the health of our oceans, coastal ecosystems and inland influences on coastal waters for improved resource management.

- Intergovernmental Oceanographic Commission International Ocean Data and Information Exchange (IODE) - an international effort to enhance marine research, exploitation, and development by facilitating the exchange of oceanographic data and information between participating member states and by meeting the needs of users for data and information products.

- Joint Commission on Oceanography and Marine Meteorology (JCOMM) - an international joint commission of the World Meteorological Organization (WMO) and the Intergovernmental Oceanographic Commission of UNESCO which coordinates, regulates, and manages a fully integrated marine observing, data management, and services system utilizing state-of-the-art technologies and capabilities.

- Open Geospatial Consortium (OGC) - an international consortium of companies, government agencies, and universities participating in a consensus process to develop publicly available interface specifications to support interoperable solutions that "geo-enable" the World Wide Web, wireless, and location-based services.

- OpenIOOS-an interoperability demonstration portal representing an effort to develop a Web Services Architecture for Ocean Observing that is enabling observing systems to move closer to the vision of 'network as platform.' This effort relies on OGC standards and is a community wide effort including research universities and several federal agencies.

- Geospatial One Stop (GOS) - a geographic information system (GIS) portal that serves as a public gateway for improving access to geospatial information and data under the Geospatial One-Stop e-government initiative.

- Global Change Master Directory (GCMD) - a long-term NASA-sponsored effort to enable discovery, access, and use of Earth science data and data related services worldwide. The GCMD offers descriptions of Earth science data sets using the Directory Interchange Format (DIF) which offers the capability to return data sets based on a user specified database query.

- Marine Metadata Interoperability Project (MMI) - an NSF funded project to promote collaborative research in the marine science domain by simplifying the incredibly complex world of metadata into specific, straightforward guidance. MMI provides advice and resources to

\section{- 2 - INTERIM DRAFT}


encourage scientists and data managers to apply sound metadata practices from the start of a project.

- NOAA IOOS Data Integration Framework (DIF) - a framework to improve the management and delivery of an initial subset of ocean observations. The DIF will establish the technical infrastructure, standards, and protocols needed to improve delivery of five core variables (seawater, temperature, salinity, sea level, currents and ocean color).

- NOAA's National Ocean Service (NOS) Data Explorer-a metadata catalog with interactive mapping tools that allow users to locate NOS products throughout the United States and its territories through a metadata catalog. Search results provide users with metadata records and links to websites with additional information.

- NOAA's Multiuse Marine Cadastre - a marine information system for the outer continental shelf and state waters. It provides direct access to authoritative marine cadastral data, and demonstrates how boundary and other data are being used to support decisions on ocean uses.

- Gulf of Maine Ocean Data Partnership (GOMODP) - a partnership to promote and coordinate sharing, linking, electronic dissemination, and use of data on the Gulf of Maine region. Through the coordinated access to the respective databases, the participants advance an integrated ocean observing system in the Gulf of Maine, promoting an understanding of the diversity and distribution of life and contributing to integrated oceans management

- Weather and Climate Toolkit, NOAA National Climatic Data Center (NCDC) - The WCT allows the visualization and data export of weather and climate data, including NEXRAD Radar, GOES Satellite, NOMADS Model and surface in-situ data. By leveraging the NetCDF for Java library and Common Data Model, the WCT is extremely scalable and capable of supporting many datasets.

- Google Search Technology, GeoSearch, Google Gadgets, Google Earth and Google Oceans Tools and services developed by Google Inc.

- COVE - a technology project with Microsoft and the University of Washington.

- OPeNDAP - Open-source Project for a Network Data Access Protocol

- THREDDS Data Server (TDS) - Thematic Real-time Environmental Distributed Data Services is developing middleware to help publish and access scientific data on the web. Built on NETCDF JAVA, THREDDS uses a browseable catalog structure to archive data sets. TDS is commonly used for hosting numerical model and satellite data.

- NOAA ERDDAP (Environmental Research Division's Data Access Program) - a web service that helps humans and computer programs download scientific data in common file formats and make graphs and maps.

- NOAA Environmental Data Connector (EDC) - a tool to connect ArcGIS tools to OPeNDAP/THREDDS catalogs and data that allows GIS users to access scientific data

\section{- 3 - INTERIM DRAFT}


- ESRI ArcGIS Server and Portal Toolkit Technology - Commercial GIS tools for desktop and server GIS and geoprocessing.

- GeoServer - open source software server written in Java that allows users to share and edit geospatial data. Designed for interoperability, it publishes data from any major spatial data source using open standards.

- NOAA National Coastal Data Development Center (NCDDC) Metadata Enterprise Resource Management Aid (MERMAid) - a tool to create, validate, manage, and publish FGDC compliant metadata.

- Coriolis - a project that includes 7 institutes in France involved in operational oceanography (Cnes, Cnrs, Ifremer, Ipev, Ird, Météo-France, Shom) and manages and distributes operational oceanography data

\section{Data Network Goals}

The goal of the Massachusetts Ocean Partnership data network is to meet user needs for access to constantly evolving data. There are many separate data sources which provide many different kinds of data. These sources must be integrated, allowing the user to discover new data and access the information in a convenient way. As new datasets are developed and old datasets become obsolete, it will be essential to continually update and monitor the database. This will be especially important in the rapid evolution of real-time observing systems where new data is coming on-line; the data network must be able to integrate new data sets automatically without architecture or client application changes.

User needs are central to the development of this data integration network; however their needs are highly varied. It is important within the data network to allow for interrogation of data sets based on user defined threshold values, the presence of indicators or the capability to design ranking schemes based on data layers. This would allow for diverse user needs to be met. It is important that a variety of different tools can access data on the network, ranging from simple web browsers for searching and previewing data, to tools such as Matlab and ArcGIS, widely used by scientists and managers. MOP maintains a data inventory in MS Access database that catalogs the data sources by topic for the user, including water column features, seabed features, habitat, archeological and cultural sites, human uses, economic valuation and climate change. This organization by user group and topic is a useful structure for planning and evaluating the data network.

\section{Data Network in Context}

Massachusetts Ocean Partnership seeks to establish a dynamic data integration network for standardized access to Massachusetts' Coastal and Ocean data. To be effective, the proposed network should assimilate with larger scale efforts such as the regional Integrated Ocean Observing System (IOOS) association to which Massachusetts belongs, the Northeastern Regional Association of Coastal Ocean Observing Systems (NERACOOS) and ultimately the National level IOOS as well. Through the use of data management best practices and interoperable standards and protocols, the MOP should recommend an implementation based on cooperative sharing of data and technologies across the state's stakeholder groups and beyond.

\section{- 4 - INTERIM DRAFT}


While data integration efforts have initially been focused on for the Massachusetts Ocean Planning Area, the ultimate goal for MOP is "to advance integrated multi-use ocean management for the purpose of ensuring healthy, resilient ocean ecosystems and thriving, sustainable marine dependent communities, industries and businesses." This goal, by definition of multi-use ocean management, expands the boundaries of the problem beyond the planning area. Ocean waters, resources, and user groups move freely in and out of the Commonwealths waters and into the watersheds. The data network and management strategy must account for this and be coordinated with and compliment regional data management efforts.

Some discussion of the other data integration efforts is valuable and necessary in order to synchronize efforts and better serve the ultimate goal of truly integrated ocean management. In order of magnitude, The Commonwealth of Massachusetts is a subset of the NERACOOS and MARCOOS regions which are a sub-set of the IOOS integration effort, which itself is the United States contribution to the Global Ocean Observing System (GOOS). It is beneficial to recognize these parallel data integration efforts and leverage lessons learned. This section will discuss a few of those networks and projects with which the Commonwealth's Data Network would coexist, support, and potentially contribute.

\section{GOOS}

The Global Ocean Observing System (GOOS) is an international program focusing on the collection and dissemination of ocean observations and derived products, including analysis, forecast, and assessment of the ocean environment. Additional elements include the development and transfer of technology to build these capabilities. The system design is scientifically based and subject to continuing review. One of the underlying philosophies of GOOS, as stated by the Intergovernmental Oceanographic Commission $(\mathrm{IOC})$, is transparent data sharing; "GOOS contributors are responsible for full, open, and timely sharing and exchange of GOOS-relevant data and products for non-commercial activities," (IOC, 1998).

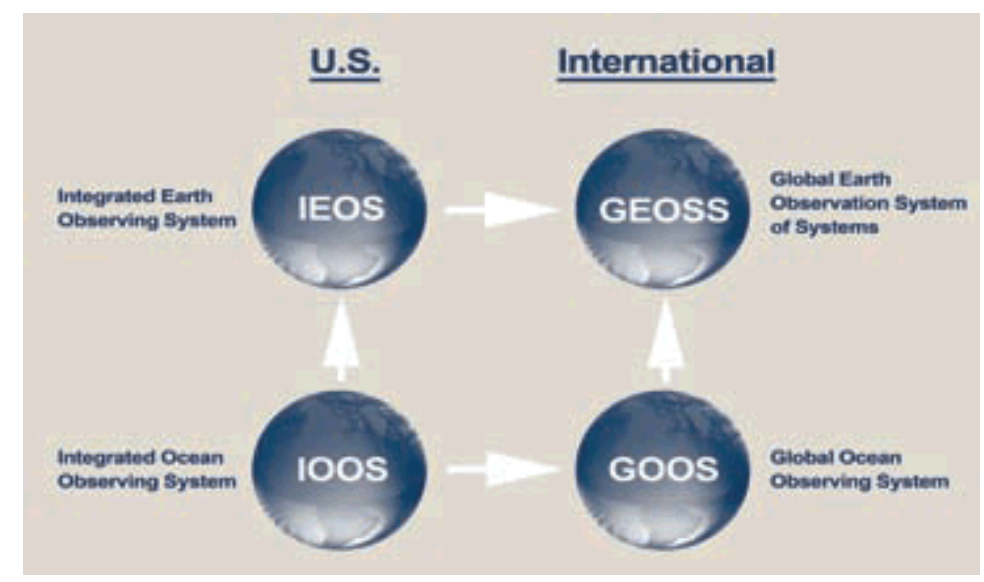

Relationships among the IOOS, U.S. IEOS and the international observing systems, GOOS and GEOSS. The IOOS is the ocean and coasts component of the IEOS and the U.S. contribution to GOOS and GEOSS.

\section{IOOS}

In 2004, Ocean.US in collaboration with federal agencies (NOAA, NASA, NSF, US Navy, EPA, USACE, USGS, MMS, and USCG), developed the first Integrated Ocean Observing System (IOOS) plan. IOOS was described as a coordinated national and international network of observations and telemetry (O\&T) elements, data management and communications (DMAC) elements, and data analyses and modeling

\section{- 5 - INTERIM DRAFT}


(DAM) elements that systematically and efficiently acquire and disseminate data and information on past, present and future states of the oceans and U.S. coastal waters to the head of tide.

The system was envisioned to aid in the achievement of seven societal goals:

(1) Improve predictions of climate change and evaluate its impact;

(2) Improve the safety and efficiency of maritime operations;

(3) Effectively mitigate the effects of natural hazards;

(4) Improve national and homeland security;

(5) Reduce public health risks;

(6) More effectively protect and restore healthy coastal ecosystems; and

(7) Enable the sustained use of ocean and coastal resources.

The new NOAA IOOS program office has re-energized the IOOS framework with a mission is to "lead the integration of ocean, coastal, and Great Lakes observing capabilities, in collaboration with Federal and non-Federal partners, to maximize access to data and generation of information products, inform decision making, and promote economic, environmental, and social benefits to our nation and the world."

Pieces of the observing infrastructure and interoperability goals have been advanced but the national funding levels are nowhere near what the design estimates suggested. In 2006, Ocean.us awarded 2 contracts, one to Raytheon and one to Lockheed Martin to provide a conceptual design for IOOS and a cost estimate. Despite the shortfall of funding (Raytheon estimated that over \$2B would be required for 2008 to meet the design requirements), many of the early goals such as the IOOS Program office, the regional associations and some portals have successfully been established. There is a strong focus on the development and implementation of standards to enhance interoperability and significant progress has been made to share observing and model data between the regional associations, the federal agencies, and the user community. 


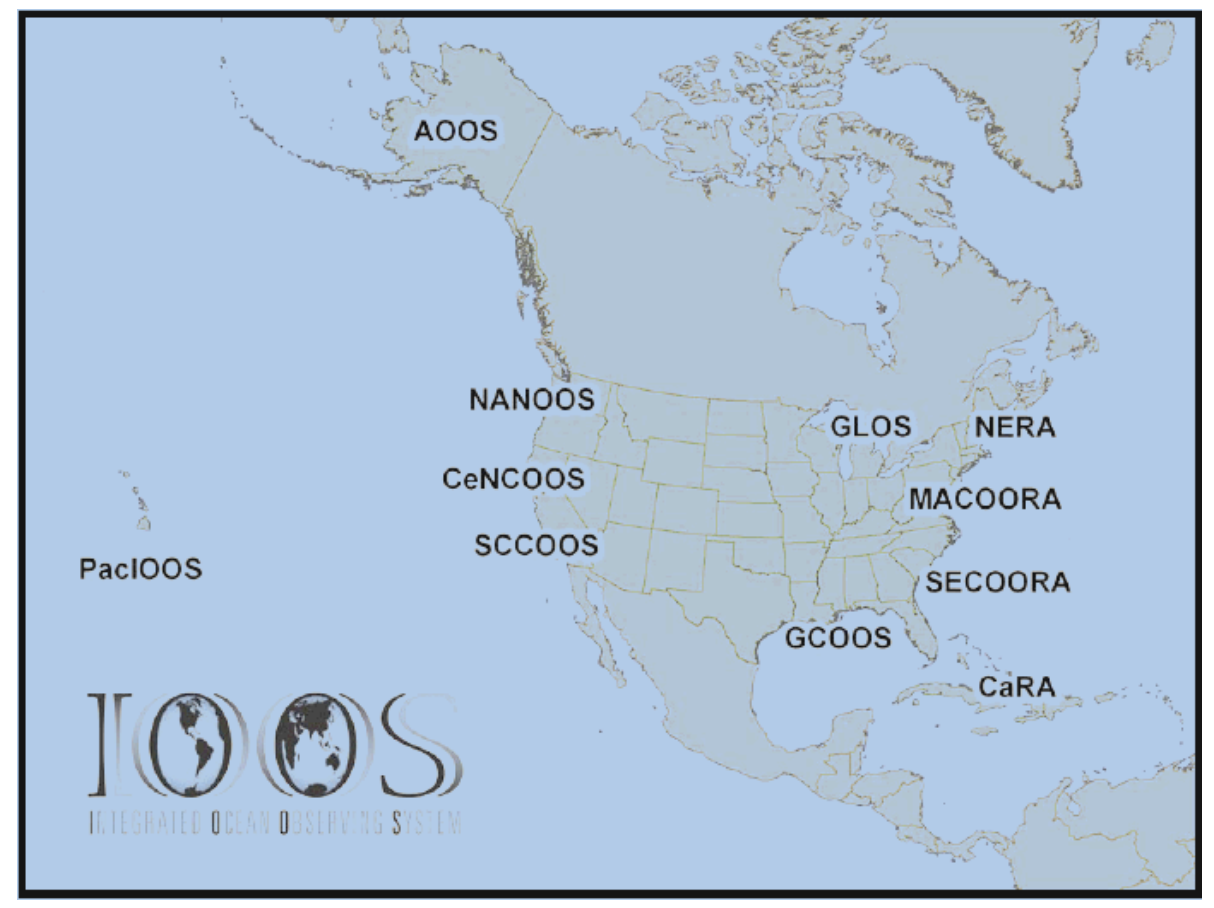

IOOS is made up of 11 Regional Associations

IOOS is made up of 11 regional associations (RAs) and these grass roots regional associations continue to show considerable advancement, initially through "plus up" federal funding and more recently through the IOOS Program Office that funds the 11 regional associations on the order of approximately $\$ 2 \mathrm{M}$ per year. The regional associations use this funding in a variety of ways including deployment of observing platforms such as in-situ buoys, high frequency radar, gliders, and for numerical modeling efforts that integrate real-time data for model forecasts for the region. Each regional association also has a data management and communications (DMAC) component that focuses on integration of the data from the different researchers in the RA.

\section{IOOS subsystems}

The architecture and goals of the IOOS and the methods for their implementation can serve as a model for the work to be done in Massachusetts. The IOOS Implementation Plan generally suggests the following: a focus on system engineering that will meet the task required, continued planning and management to allow for adjustments and amendments to original plans, and coordinated regional activities that support the larger effort. The Massachusetts data network is a regional activity which should coordinate with IOOS, while using the same principles in its strategic planning.

The IOOS implementation plan and the program itself focus on a number of discrete components or subsystems, the Observing Sub-System, the Data Management and Communications Sub-System, the Modeling Sub-System and an Education component. By isolating the sub-systems, independent parallel work allows for the goals to be met in shorter timespan. The generic goals are to integrate existing assets, improve those assets and develop new assets through enhancements and research.

\section{- 7 - INTERIM DRAFT}




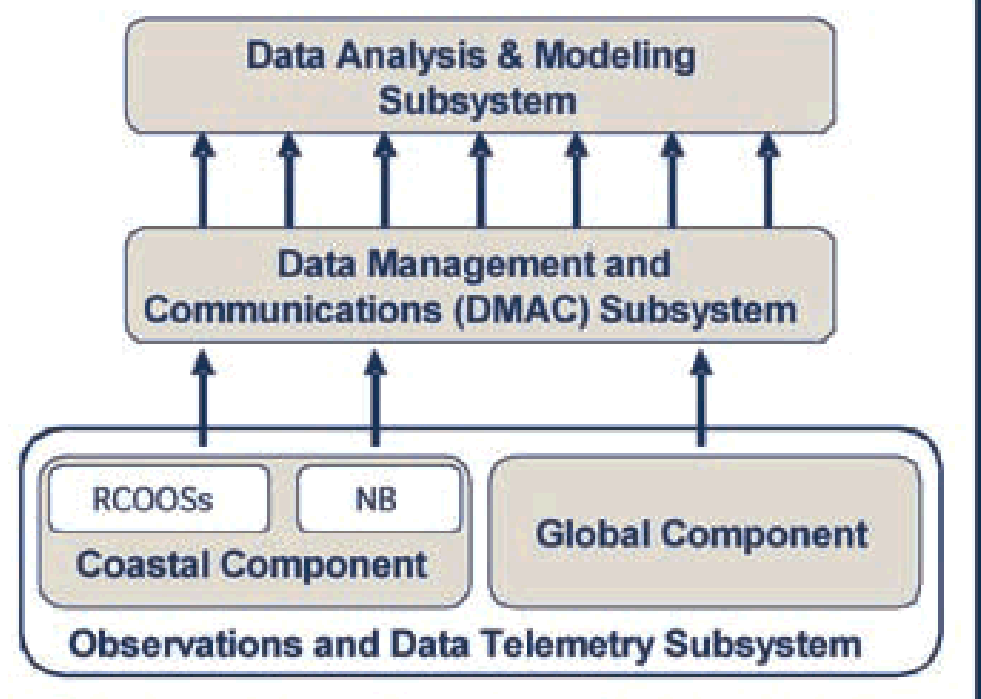

IOOS Component Schematic (courtesy NOAA IOOS)

The observing subsystem consists of global and coastal components with the latter broken down into a National Backbone (NB) for the Nation's Exclusive Economic Zone (EEZ) and Regional Coastal Ocean Observing Systems (RCOOSs) to address regional and local needs. The integrating engines are the DMAC and modeling subsystems. The NB provides data and information required by federal agencies and most, if not all, Regional Associations. RCOOSs contribute to the NB and are tailored to the data and information needs of each region.

IOOS has defined their Data Integration Framework (DIF) which they are actively implementing through the establishment of the technical infrastructure, standards, and protocols needed to improve delivery of these 7 initial "core ocean observation variables":

- Sea Temperature

- Salinity

- Water/Sea level

- Currents

- Ocean color

- Waves

- Surface Currents 

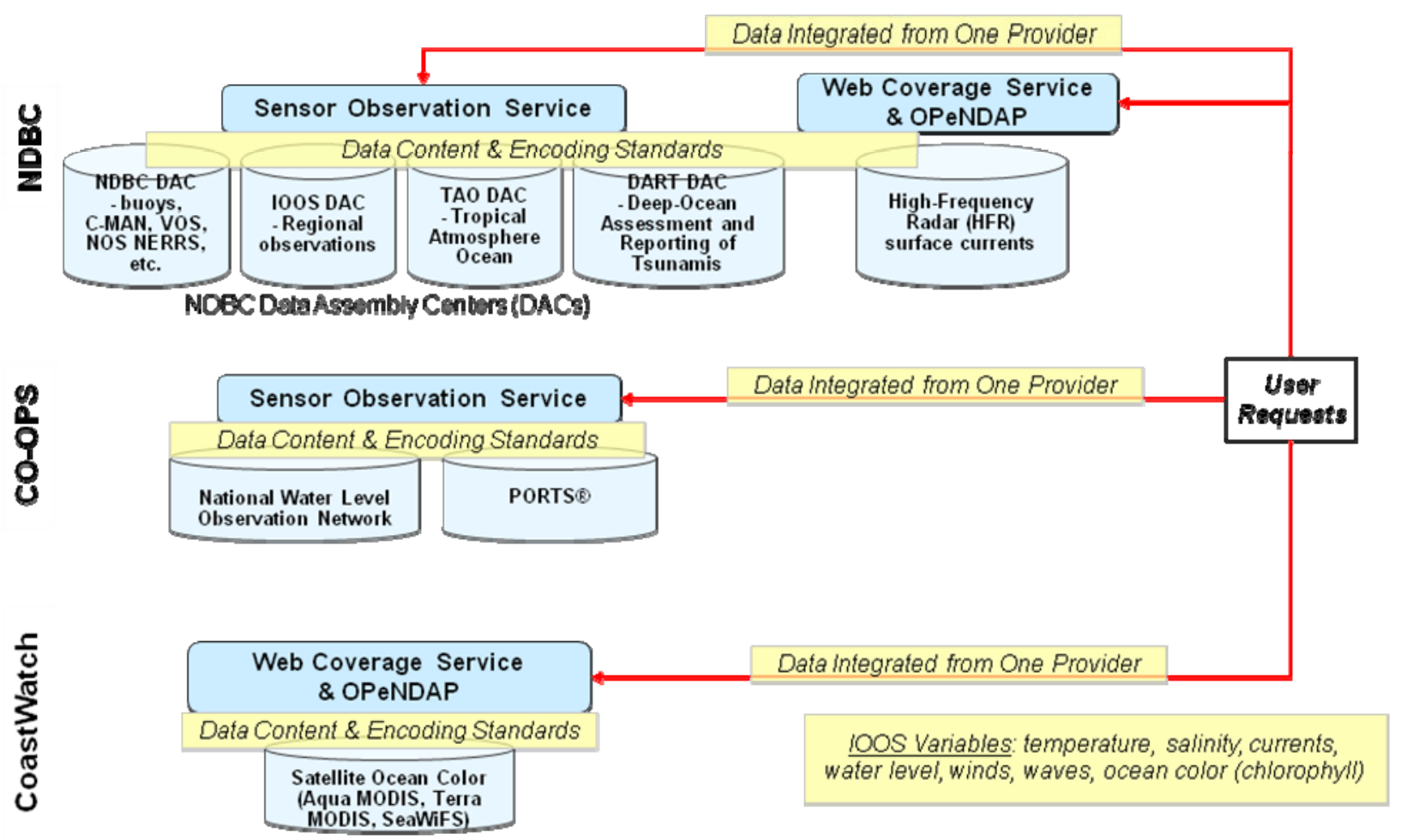

IOOS Data Integration Framework (DIF) Data Provider Status (courtesy NOAA IOOS)

It will be important that a data network in Massachusetts uses similar architecture, standards, and formats as the DIF to allow for regional integration and consistency with the regional associations (RAs) as they are in the process of implementing the DIF. The Massachusetts data network does not need to mirror exactly the DIF, but by using key components of the DIF, the network can share data seamlessly.

\section{Neracoos}

The Northeastern Regional Association of Coastal Ocean Observing Systems (NERACOOS) is the IOOS regional association extending from Connecticut and Long Island Sound northward to Maritime Canada. The region includes the coastal waters of Maine, New Hampshire, Massachusetts, Rhode Island, and Connecticut. NERACOOS has a number of partners, primarily academic institutions that perform ocean observing and modeling as well as a data management team that focuses on the integration and distribution of the data. NERACOOS also has the benefit of being able to leverage the work done by GoMOOS (Gulf of Maine Ocean Observing System), a regional coastal ocean observing system that has focused on ocean observations, data management, and interoperability for many years.

The Massachusetts data network can benefit from the NERACOOS work in several ways:

- as a framework to connect to and collaborate with researchers and data providers in the region;

- through coordination on data standards, appropriate data cataloging, and methods to expose native data; and by

- leveraging technologies that deliver observation data in a number of standardized outputs, and existing outreach and education efforts.

\section{- 9 - INTERIM DRAFT}


NERACOOS is currently going through a restructuring exercise with new management, a new board and new strategic and data management committees.

\section{GoMODP}

The Gulf of Maine Ocean Data Partnership was established to promote uniform management and use of data in the Gulf of Maine region. The GoMODP is focused on environmental data authority, discoverability, accessibility, and interoperability. The GoMODP participants have made a commitment to coordinate their efforts to best take advantage of the significant data in their respective databases.

GoMODP has a strong relationship with NERACOOS and its members include governmental agencies, intergovernmental organizations, and nongovernmental organizations, including academic, research, and other nonprofit entities. Through coordinated access to shared data resources, the GoMODP participants hope to achieve a fully integrated ocean observing system and ultimately contribute to integrated oceans management. The GoMODP has already made significant contributions in the science community by providing tools for cooperation and mutual data access to models and observations. The GoMODP is a great resource to assist in organizing and processing data from the science community for the data network.

\section{OOI}

The National Science Foundation (NSF) Ocean Sciences Division has developed the Ocean Observing Initiative (OOI) which combines state of the art ocean observation systems with sophisticated cyberinfrastructure $(\mathrm{Cl})$ to support scientific research. OOI focuses on advancing technology and has successfully implemented a number of pilot projects. As OOI develops, more integration is expected with the IOOS initiatives. A number of cross fertilization projects between OOI and IOOS are already underway.

It is important that a data network continues to evaluate emerging technologies, especially related to the cyber-infrastructure evolving in the OOI program. The OOI effort to develop cloud computing is a very promising technology for building highly scalable networks and a number of $\mathrm{Cl}$ pilot projects are on -going that could lead to significant improvements in performance related to large scale science data collection and distribution. The ability for an ocean data network to be aware of these efforts and build network components that can leverage these efforts will be important, especially as the volume of integrated observing data increases. 


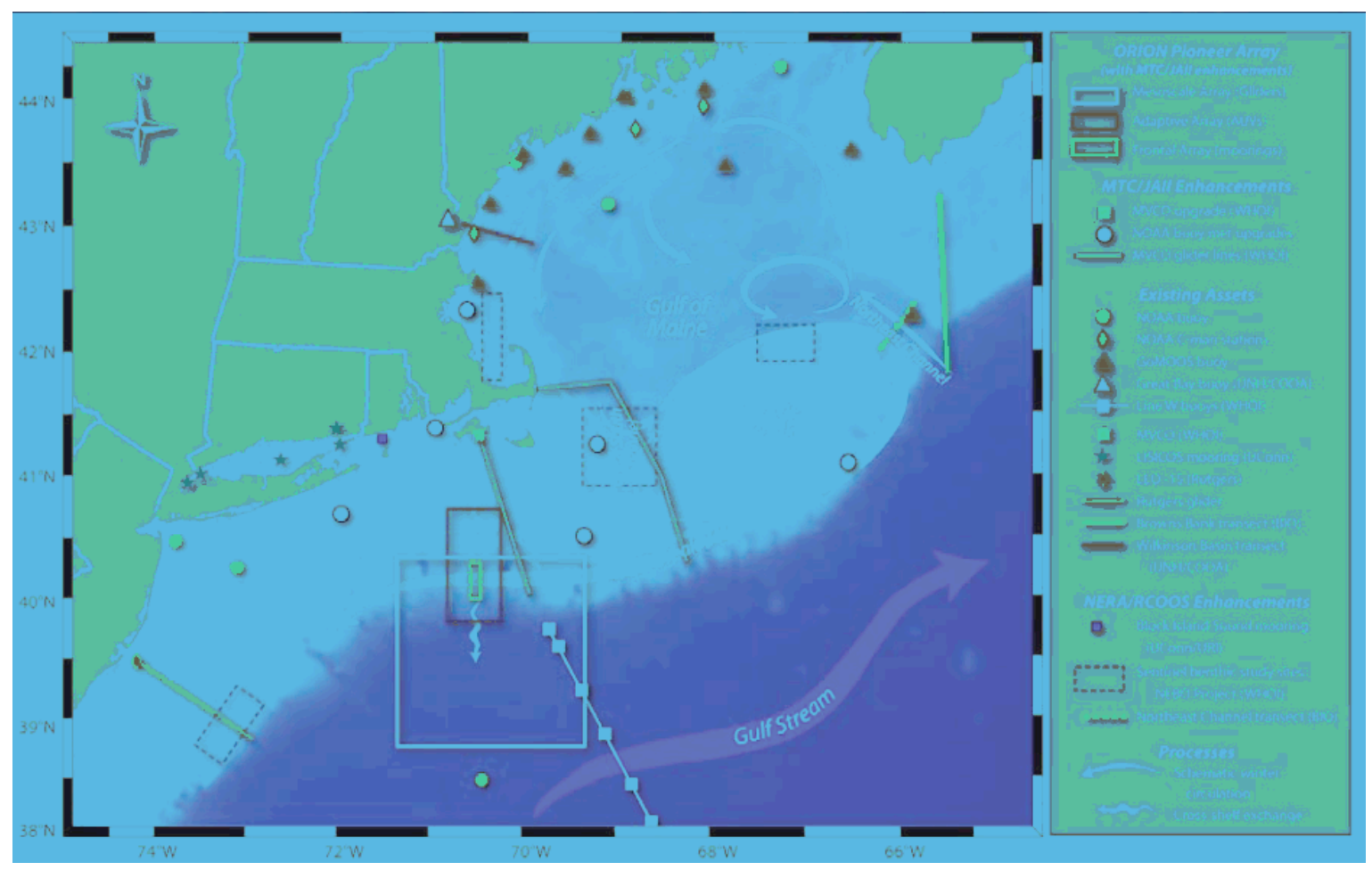

Vision for combined OOI and IOOS assets (courtesy NSF)

\section{User Needs}

Meeting user needs is a main driving force for the development of the data network. In order to fully understand user needs, each user or user group should be identified and interviewed, convened, or surveyed.

Resource managers are a primary user group that the Massachusetts Ocean Data Network should serve. The data needs of resource managers are well represented in the six reports from the Ocean Management Planning work groups. Those six reports cover data related to the following topics:

- Renewable energy

- Habitat

- Fisheries

- Sediment

- Transportation, navigation and infrastructure

- Recreational and cultural services

Each work group report can be used to understand the data necessary to manage ocean uses, resources, and services - as well as to better understand the potential needs of other related user groups. Summaries of work group reports are outlined below and are followed by a discussion of a preliminary user needs assessment. 


\section{Work Group Summaries}

The mission of the Ocean Management Planning work groups was to collect and synthesize data that support the identification of locations in the planning area of importance or special concern to the group. Each group also identified data and information gaps, and made recommendations about future data collection and management. The groups started by defining information it considered "important" to their topic, and collected and reviewed relevant data to determine data quality and data gaps. The available data was then analyzed and synthesized using a classification system specific to each work group that identified areas of importance. The groups then produced images identifying areas that were categorized as sensitive or important. This process provides valuable background information to the MOP and data network developers about the data collection, visualization, and analysis needs of resource managers and other data network user communities.

\section{Fisheries Work Group}

The Fisheries Work Group report identifies locations in the ocean management planning area of high importance to fishery activity and fishery resources, and identifies future data management needs.

Data was collected from fishery dependent and independent sources. Commercial fishery data was obtained via the National Marine Fisheries Service (NMFS) and the MA Division of Marine Fisheries (DMF). Recreational fishery data was collected from the Marine Recreational Information Program (MRIP) which conducts intercept and random telephone surveys to estimate recreational saltwater catch and effort. The most significant data obtained from a non-fishery source, was from the DMF Resource Assessment Bottom Trawl Survey.

The fisheries work group also made recommendations about future data collection and management improvements. Two primary recommendations include:

- Implementing "Comprehensive Fishermen Trip-Level Reporting" to fill data gaps and avoid duplicative reporting, and

- Upgrading recreational catch and effort surveys to include better spatial information on where trips occur

\section{Habitat Work Group}

The Habitat Work Group report identifies sensitive, critical or protected habitats in the ocean management planning area and discusses subject areas and geographic locations that lack complete data.

The Habitat work group identified three different types of habitat data:

- Areas and resources with special legal protection;

- Habitat critical to or providing specific life stage support for important species (biotic data); and

- Unique and/or sensitive habitats as indicated by abiotic parameters

The majority of the data providers were Massachusetts regulatory agencies and environmental organizations, including the MA Department of Environmental Protection, Mass Audubon Society, MA Division of Marine Fisheries, and the MA Office of Coastal Zone Management.

\section{- 12 - INTERIM DRAFT}


The group also identified several other datasets in development that will provide use in classifying the habitats of the ocean management planning area. These data include outputs from the North East Coastal Ocean Forecast System (NECOFS) and extractions from the Mass Audubon Bird Observer Database. Additional data identified as potentially helpful to the habitat working group in the future include the CZM and USGS Seafloor Mapping Cooperative's characterization of the seafloor, which at present does not have enough spatial coverage to be useful in large area planning.

\section{Ocean Recreational and Cultural Services Work Group}

The Ocean Recreational and Cultural Services Work Group report determines places in the MOP planning area that are of special cultural, visual and recreational value to society.

Most of the data used to support the Ocean Recreational and Cultural Services Work Group report were GIS layers, from varying sources. When determining historic places of note, the work group used layers that depicted archaeological resources and ship wrecks. Sites of value for ocean recreation included vessel-based recreation boat launches, SCUBA diving sites, outstanding resource waters, mooring fields, marina, artificial reefs and recreational boating areas. Coastal access, marine beaches and scenic landscapes were identified as public viewing sites.

It was determined that although historical data exists in the planning area, the reliability of the information is questionable. Therefore, an updated database would be useful to the workgroup. In addition, additional data regarding vessel-related recreational activities and describing scenic viewing services would both be helpful.

\section{Transportation, Navigation and Infrastructure Work Group}

The Transportation, Navigation, and Infrastructure Work Group report identifies locations in the ocean management planning area where transportation and marine navigation occurs, as well as areas where the infrastructure supporting existing uses are currently located.

Most of the data utilized by the work group are available through MORIS (Massachusetts Ocean Resource Information System). The layers display the locations of utility, sewer and cable lines, anchorage sites, precautionary or prohibited areas, ship channels/fairways, disposal and spoil areas, MWRA outfall diffusers, artificial reefs, and the footprints of proposed tidal energy projects. The sources not found in MORIS describe ferry routes and navigation aides.

The work group pointed to a couple of topic areas where data gaps exist. More information regarding recreational and commercial vessel patterns and improvements to existing MORIS layers showing cable lines would both be useful.

\section{Sediment Work Group}

The Sediment Work Group report identifies the different sediment types within the ocean management planning area and determines their chemical and physical properties.

This workgroup leveraged data available through the MORIS system in GIS format, including the ocean management planning area boundaries and grids. Additional layers not obtained through MORIS describe the bathymetry, sediment size, potential sediment resources, and dumping grounds throughout the planning area. 
The sediment work group listed a number of datasets that would be useful should they be converted to a GIS format. These data sets consist mostly of core data sampled at specific locations and a USGS map of physiographic zones. Physiographic or geomorphic zones are subdivisions based on terrain texture, rock type, and geologic structure and history. In addition, the work group identified several topic areas where no data or limited data exists. These relate to sediment transport, contaminated sediment, wave modeling, bottom and sub-bottom type categorization, and acoustic seafloor mapping.

\section{Renewable Energy Work Group}

The renewable energy work group report identifies locations in the planning area that would be appropriate for renewable energy development, specifically tidal, wave and wind energy.

The data explored for tidal energy projects came primarily from previous technical reports published by EPRI and Navigant Consulting. Wave energy was briefly explored through technical reports, but wave energy development is not foreseen in the next 10 years in the planning area due to a limited energy resource. Wind energy was researched using technical reports, and datasets describing wind speed, water depth, seabed geology, wave height, electrical transmission lines and port infrastructure collected from a variety of sources.

The energy work group recommended certain locations where more information should be gathered to assess suitability for renewable energy projects, including for example, additional research in Buzzards Bay for possible tidal energy projects. The report also indicated that more research should be conducted to understand ports that could be utilized as a construction base for renewable energy facilities. Lastly, as renewable energy technology is evolving rapidly, the data and derived maps must be updated regularly once the network is online.

\section{Method of User Need Assessment}

User needs help to define the role of the data integration network for Massachusetts coastal and ocean data. Understanding the role of the data integration network is a challenging but essential task.

In addition to these six work groups, the EOEEA data team, the Public, and regulators are also important users. Utilizing the meeting minutes from the Science Advisory Council, each of these nine potential user groups were analyzed in the Assessment of User Interests and Goals Table. For each user their interest, environmental policy goal, data access needs and present data accessibility were identified. The goals of each user can be used to evaluate the priorities of the data integration network design.

The clear challenge after assessing user needs is striking a balance between the wide varieties of interests. Policy makers grapple with this issue on a daily basis. Using indicators and ranking schemes can help simplify a large amount of information into a useful form. For example the concept of critical load was developed in the 1970s to address the problems in addressing pollution control. Mapping the critical loads can show where pollution loads are above the threshold values.

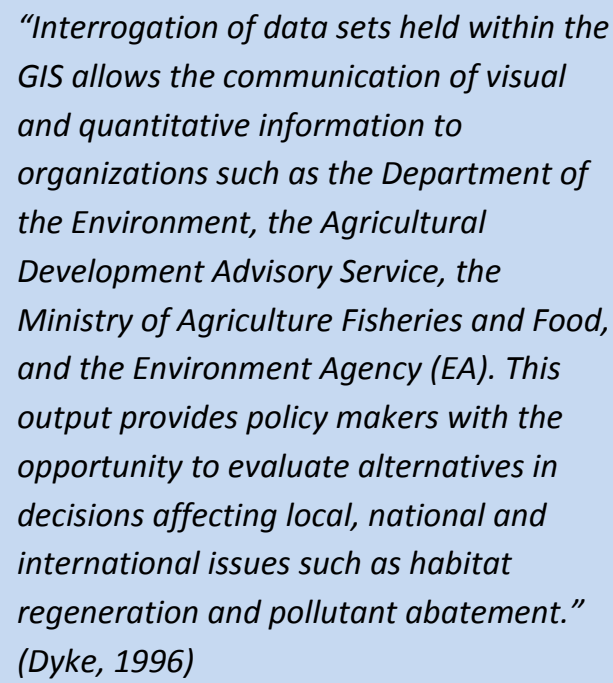

"Interrogation of data sets held within the

\section{- 14 - INTERIM DRAFT}


Another example of using indicators and ranking schemes is being used in Rhode Island as part of its Ocean Special Area Management Plan (SAMP). Investigators at the University of Rhode Island have developed the Technology and Energy Resource Based Screening Analysis that allows identification of potential wind farm sites in RI coastal waters for monopile, jacket, and other wind turbine foundation systems. The analysis is based on an estimate of Technical Development Index (TDI) which is defined as the ratio of the Technical Challenge Index (TCl) to the Power Production Potential (PPP). The SAMP study area is divided into discrete grids (approximately $100 \mathrm{~m}$ resolution) and an analysis performed for each grid. Low values of TDI indicate favorable potential sites for wind farms.

Generating a data integration network that can rapidly integrate new data sets and apply rules-based calculations and rankings as well as visualization of a wide variety of data including indicator organisms or threshold values would be very valuable and could meet the diverse needs of the stakeholders.

In conclusion the user needs; (1) are central to the development of the data integration network and (2) can be used to assess the value of various design options. Generally the diversity of needs can be met by allowing for client software applications that focus on appropriate data for specific user groups and allow interrogation of data sets based on user defined threshold values, the presence of indicators or the capability to design ranking schemes based on data layers. 


\begin{tabular}{|c|c|c|c|c|c|}
\hline User & Interest & Environmental policy goal & Data access needs & Present data accesibilty & $\begin{array}{l}\text { Course of } \\
\text { solutions }\end{array}$ \\
\hline $\begin{array}{l}\text { Ocean } \\
\text { Renewable WG* }\end{array}$ & $\begin{array}{l}\text { Promote Renewable } \\
\text { Energy }\end{array}$ & $\begin{array}{l}\text { Realize renewable energy } \\
\text { projects in mass planning area }\end{array}$ & $\begin{array}{l}\text { Identify optimum placement of renewable energy } \\
\text { projects }\end{array}$ & Suitability ranking schemes and individual feasibility studies & \\
\hline Habitat WG* & Protect the Habitat & $\begin{array}{l}\text { Balanced ecosystem for all } \\
\text { species }\end{array}$ & $\begin{array}{l}\text { Identify, view and monitor "endangered habitat } \\
\text { areas" and analyze data for potential habitat }\end{array}$ & Data in different formats, scales, and from disparate sources & \\
\hline $\begin{array}{l}\text { Comm/Rec } \\
\text { Fishing WG* }\end{array}$ & Maintain fisheries & Sustainable fisheries & $\begin{array}{l}\text { View the probabilities of where and when they } \\
\text { could catch fish; understand impacts on } \\
\text { commercial and recreational fisheries }\end{array}$ & $\begin{array}{l}\text { Digitized maps showing distribution of } 22 \text { most important } \\
\text { species \& areas of importance }\end{array}$ & \\
\hline Regulatory & $\begin{array}{l}\text { Maintain appropriate } \\
\text { policies and regulations }\end{array}$ & $\begin{array}{l}\text { Balanced use of ocean } \\
\text { resources and services }\end{array}$ & $\begin{array}{l}\text { View most recent data to asses if policies are } \\
\text { working or if they need revision and understand } \\
\text { existing regulatory framework }\end{array}$ & $\begin{array}{l}\text { NOAA multipurpose marine cadastre and legislative atlas } \\
\text { provide some information }\end{array}$ & \\
\hline $\begin{array}{l}\text { Sediment Mgt } \\
\text { WG* }\end{array}$ & $\begin{array}{l}\text { Understand sediment } \\
\text { patterns and manage } \\
\text { dredged material }\end{array}$ & $\begin{array}{l}\text { Achieve balanced and } \\
\text { sustainable solutions to } \\
\text { sediment-related needs }\end{array}$ & $\begin{array}{l}\text { Capability to do site specific sediment analysis that } \\
\text { can be nested into a statevide model. }\end{array}$ & $\begin{array}{l}\text { Missing information on contaminated sediments. Dredging } \\
\text { project data, DMF lobster habitat data and Neptune data } \\
\text { need to be integrated. Suitability rankings identified, but } \\
\text { need more definition. SPI has profile data. MMRA has hard } \\
\text { bottom information. }\end{array}$ & \\
\hline $\begin{array}{l}\text { Trans/ } \\
\text { Nav/Infrastruc } \\
\text { WG }^{\star}\end{array}$ & $\begin{array}{l}\text { Keep navigation } \\
\text { transportation lanes } \\
\text { open and safe }\end{array}$ & Safe and open navigation & $\begin{array}{l}\text { View traffic levels and ensure competing uses do } \\
\text { not interfere with navigation, using real time data } \\
\text { from ships with tracking devices. View real time } \\
\text { bathymetry data for dredging needs. }\end{array}$ & $\begin{array}{l}\text { NOAA nautical charts, Missing Army Core sites, other misc } \\
\text { missing data, Unclear vessel routes for ferries, commercial } \\
\text { and fisherman vessels }\end{array}$ & \\
\hline $\begin{array}{l}\text { Oultural/ Social/ } \\
\text { Rec WG* }\end{array}$ & $\begin{array}{l}\text { Protect recreational and } \\
\text { cultural senvices and } \\
\text { activities in the region }\end{array}$ & $\begin{array}{l}\text { Clean and pleasant place for } \\
\text { recreation and tourism }\end{array}$ & $\begin{array}{l}\text { View layers on recreational usage (boating traffic, } \\
\text { kayaking, wind surfing, sailing) and cultural sites. } \\
\text { Visualizations of ocean views }\end{array}$ & $\begin{array}{l}\text { Existing data on recreational and cultural resources of little } \\
\text { use. Missing information on scenic value of the ocean. } \\
\text { Need Paleo Landscapes mapping (show no shipureck areas } \\
\text { and seabed characteristics) and get Native American tribes } \\
\text { input. }\end{array}$ & \\
\hline $\begin{array}{l}\text { EOEEA Data } \\
\text { Team }\end{array}$ & $\begin{array}{l}\text { Provide portal to } \\
\text { centralize data and } \\
\text { resources }\end{array}$ & $\begin{array}{l}\text { Easy, Fexible, Robust data } \\
\text { portal for all users }\end{array}$ & $\begin{array}{l}\text { Centralized data portal that meets all users needs } \\
\text { and is easily maintained }\end{array}$ & $\begin{array}{l}\text { MORIS database that "becomes impractical when number of } \\
\text { data layers grow into thousands" }\end{array}$ & \\
\hline $\begin{array}{l}\text { Public, Other } \\
\text { Stakeholders }\end{array}$ & $\begin{array}{l}\text { Maximize public good } \\
\text { and welfare enjoyment } \\
\text { of Ocean }\end{array}$ & $\begin{array}{l}\text { Healthy ocean and pristine } \\
\text { recreation environment }\end{array}$ & $\begin{array}{l}\text { See status of water quality at beaches, view photo } \\
\text { renderings of potential offshore wind, view basic } \\
\text { information about ocean resources }\end{array}$ & $\begin{array}{l}\text { 14+ various portals used to display a wide range of } \\
\text { information (e.g. MORIS, MassGIS) }\end{array}$ & \\
\hline
\end{tabular}

Assessment of Users Interests and Goals Table 


\section{Metadata - Searching the Network}

One of the most important aspects of any data network is the ability to quickly and efficiently discover applicable and relevant data. In order to be successful at this, all of the data in the repository needs to be cataloged in a similar fashion. The existing standards and the challenges they pose will be discussed in the section on Catalogs and formats. Following this in the metadata and discoverability section, the needs of the data network and possible improvements in metadata management are outlined.

Metadata represents the who, what, when, where, why and how of the resource, it is "data about the data". Geospatial metadata are used to document geographic digital resources such as Geographic Information System (GIS) files, geospatial databases, and earth imagery. A geospatial metadata record includes core library catalog elements such as Title, Abstract, and Publication Data; geographic elements such as Geographic Extent and Projection Information; and database elements such as Attribute Label Definitions and Attribute Domain Values. The Federal Geographic Data Committee (FGDC) describes metadata as "a file of information, usually presented as an XML document, which captures the basic characteristics of a data or information resource."

\section{Catalogs and Formats}

The FGDC standard is one of the widely used standards by which metadata is created and stored by entities that are considered metadata repositories. Two significant examples of these repositories are the NASA Global Master Change Directory (GCMD) [gcmd.nasa.gov], which uses the Data Interchange Format (DIF) and the Geospatial One Stop [www.geodata.gov], which uses the FGDC format. The standards differ in content and naming conventions for labeling metadata. It is important that the generic elements that make up the attributes in the metadata records be available in a manner that allows easy extraction, searching and cataloging. Below are the mandatory and conditional elements of the two standards discussed above. One standard cannot be translated into another standard easily since the mandatory and conditional elements differ. In addition, the naming schemes of the elements require some background understanding. Ideally a first time user should be able to intuitively understand how to search, read and create metadata. With the existing system of multiple metadata formats this is not possible. 
GCMD

Mandatory Elements:

Entry ID

Entry Title

Parameters (Science Keywords)

ISO Topic Category

Data Center

Summary

Metadata_Name

Metadata_Version
Conditional Elements:

Data Set Citation

Personnel

Instrument

Platform

Temporal Coverage

Paleo-Temporal Coverage

Spatial Coverage

Location

Data Resolution

Project

Distribution

Data Set Language

Data Set Progress

Related URL

DIF Revision History

Keyword (Ancillary Keyword)

Originating Center

Multimedia Sample

References(Publications)

Parent DIF

IDN Node

DIF Creation Date

Last DIF Revision Date

Future DIF Review Date

Privacy Status

GCMD Data Information Format Fields 


\section{Geospatial One Stop}

Mandatory Elements:

Dataset title

Dataset reference date

Dataset language

Dataset topic category

Abstract

Metadata point of contact

Metadata date stamp

\author{
Conditional Elements: \\ Dataset responsible party \\ Geographic location by coordinates \\ Dataset character set \\ Spatial resolution \\ Distribution format \\ Spatial representation type \\ Reference system \\ Lineage statement \\ On-line Resource \\ Metadata file identifier \\ Metadata standard name \\ Metadata standard version \\ Metadata language \\ Metadata character set
}

FGDC - ISO 19115 Core Metadata Elements

\section{Metadata and Discoverability}

The data included in the MOP data network must have metadata in order to appropriately catalog the assets. The assets may range from geospatial data to reports to observation and modeling systems. The assets may originate from a diverse provider group that may include federal agencies and independent academic research. As a result, it will be important to leverage the existing infrastructure and extend it in a manner that will offer flexibility and scalability.

The interface to the data must be intuitive, and offer various means by which information can be filtered and isolated. Currently, the Massachusetts Geographic Information System (MassGIS) has a protocol in place by which metadata records are established and made available to various metadata repositories. As new sources are added to the MassGIS SDE database, metadata records are created to comply with the FGDC standards. The FGDC metadata records are subsequently loaded into a "Web share" folder where they are made available to the Geospatial One Stop (GOS) harvesting application. A significant number of these records have not been accepted by the GOS application. The reason behind the rejections has not been investigated as yet.

The MassGIS records are also submitted to the Global Change Master Directory (GCMD). The FGDC XML metadata are converted manually to the GCMD DIF format. The data is sent to a person at NASA who processes the FGDC file, makes some small changes, including adding the Massachusetts Data tag and converts the elements to the DIF format for inclusion in the GCMD. The GCMD plans to implement a harvesting application for the data files located in the Web share folder in the future. 
MassGIS are strong proponents of metadata, specifically using the FGDC standard and this is common for GIS data managers. The adoption of metadata generation within the science user community is not as widespread, in fact, their attitude towards metadata implementation has been described as one of "loathing", so the data network should encourage metadata, but a pragmatic approach must be taken to allow discovery and integration of data that may not have fully populated metadata records.

One possible addition to metadata, or alternative to metadata when there is none, has been described as a "Datapedia" solution (Peter Cornillon, OPenDAP), whereby a Datapedia URL (similar to Wikipedia) is connected to all data sources and this allows data providers and, more importantly, data users to comment on their experience using the data. Although this concept may be considered a violation of quality control standards and the traditional metadata paradigm, it is one that would be used and useful. A recent example of how this could be used was a search \& rescue case where the U.S. Coast Guard used a NOAA ocean forecast and observed that the surface currents near the Gulf Stream were very strong. By talking to the NOAA forecasters, we were able to determine the cause. This information could have been entered into the Datapedia page for this data product and accessed by other users. The data provider could also provide comments and updates as the model changes to address specific issues. Handling this type of issue with traditional metadata is very difficult.

Whether data has complete metadata records or not, the data is of little value if users cannot find or discover it. In fact, this may be one of the greatest challenges facing the community as huge amounts of disparate data comes on-line in different formats. We will make recommendations on using Web 2.0 technology and expanding beyond the defined metadata searching in use by GIS and science users (GCMD/GOS).

\section{Data Categories}

For the data network design, 4 main classes of data will be considered:

\section{- Traditional static geospatial (GIS) data}

This is typically time-static data with GIS features (points, lines, polygons) and grids (rasters), it may include terrestrial, coastal and ocean features. Examples of this data could include polygons of fishing areas, coastline details, seasonal whale observations, sediment classifications, and satellite imagery.

In general, we see that most of this type of data will transport through MassGIS and their GeoServer, and can be available in a variety of formats including KML, WMS and SHP. This makes this data group relatively straightforward to integrate. However, getting new data into the MassGIS system can be time consuming, and requires coordination with MassGIS staff. It should not be underestimated how time consuming this may be, especially to meet the metadata requirements.

There are also vast amounts of GIS data available from other sources (Google, NOAA, ESRI, USGS, state agencies, etc.), the standards are well defined and interoperable through the use of OGC standards and widely used GIS formats make this category of data readily integratable. 


\section{- Gridded data}

In this category, we are considering ocean and coastal observing data from satellites, radar, and models. The grids may be rectangular, triangular, orthogonal, or some other form of unstructured grid. The data is generally time-varying and may have time spans ranging from hours to decades; the data can be enormous.

We do not recommend that this data transits through MassGIS and we see the need for a data network to access this data through distributed servers. This requires that the servers have some standard suite of web services or data protocols available for interoperability and integration.

\section{- Point Observation Data}

This is typically data from in-situ observing systems such as current meters, met stations, water quality stations, wave buoys, drifting buoys, gliders, stream gauges, and sampling stations. The data is generally time-varying.

A number of efforts are operational or maturing to integrate regional and national data through NOAA, EPA, USGS, and other federal agencies. This data is often also available from regional observing systems, but we believe a focus on connecting to the federal systems that store regional observations is most efficient.

\section{- Informational Data}

This data category includes non geographic data sources. Examples include PDFs, reports, images, websites, and spreadsheets. These data can be "geotagged" through metadata information and can provide supporting data for decision makers.

\section{Data Categories Discussion}

\section{Regional GIS Data}

The Master Data Inventory that MOP developed with Stone Environmental is stored in a Microsoft Access database and currently provides the details of 1,615 data sets. It is expected that the database will continue to expand as new data sources are identified. The data topics are primarily focused on human impacts, biology, geology and water properties in the MOP planning area, although many of the datasets have a much larger spatial extent. They are grouped into categories regarding fish (19\%), other animals (24\%), fisheries (6\%), human threats (29\%), and the benthic, coastal and oceanographic environment (22\%).

Approximately $75 \%$ of the data entries are in ESRI or shapefile format, while the remaining $25 \%$ are PDFs, reports, images, websites, and spreadsheets. The datasets come from 137 sources, $74 \%$ are without metadata. The majority of the data is either current or published after the year 2000 . There are some limitations to this data. Some of the datasets have restricted access or use policies. There is only limited information for datasets provided by MassGIS, MORIS and the Nature Conservancy project, however, contact information is provided for most of the datasets should further inquiry be necessary.

We recommend that selected data from this inventory, identified as a priority by the user groups, be integrated into the MORIS GIS system.

\section{- 21 - INTERIM DRAFT}




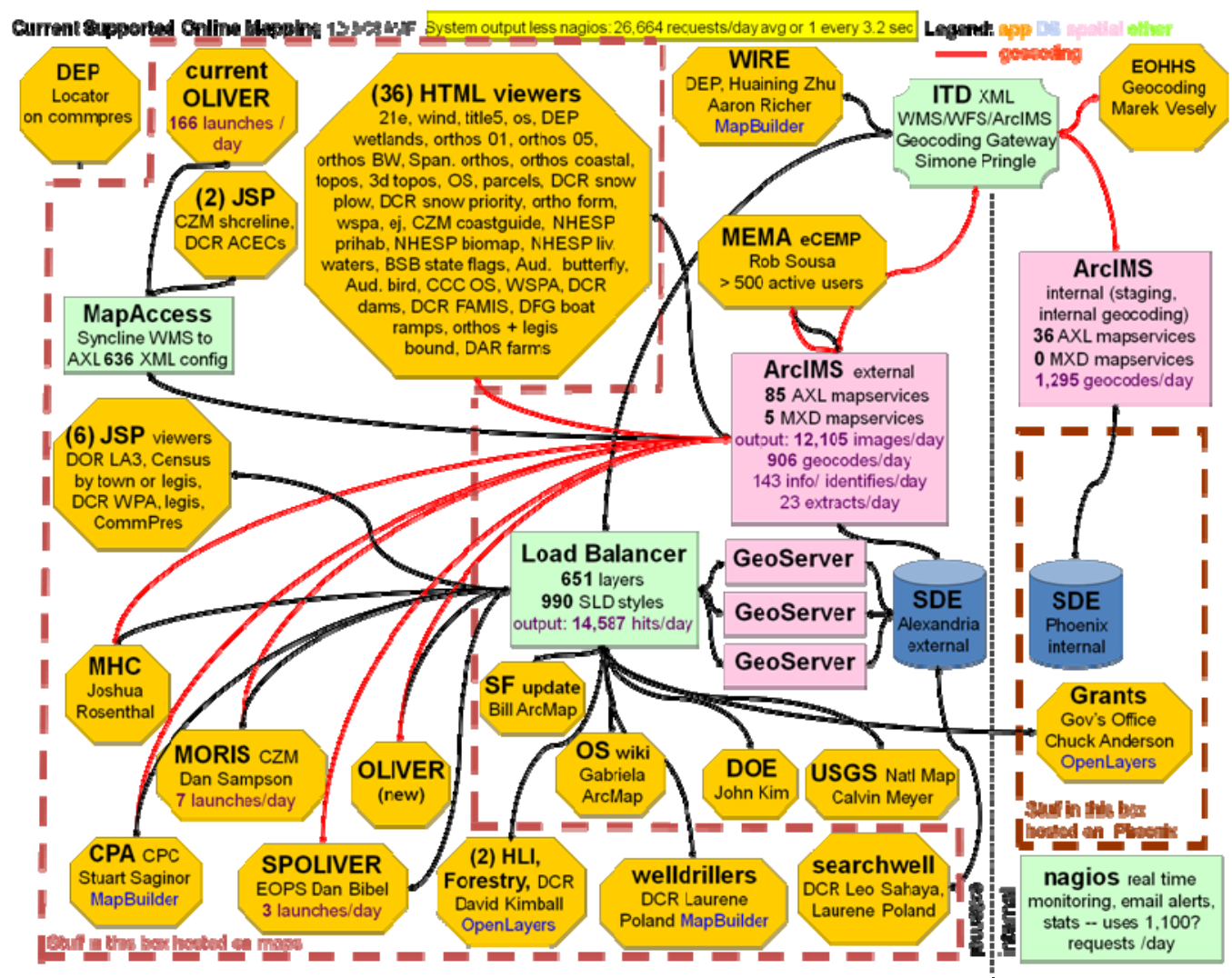

MORIS Architecture (MassGIS)

The staff at MassCZM and MassGIS were generous with their time and provided enormous amounts of information on their sophisticated MORIS system. MORIS stores, displays and distributes a vast amount of geospatial data. The system is based on 2 main pieces of technology - Geoserver and ESRI SDE. SDE is used to provide geospatial connections to the data stored in a relational database. Geoserver publishes this data, and software clients MORIS and OLIVER allow users to access the data. MORIS is used to display spatial data pertaining to the Massachusetts coastal zone. Users can interactively view various data layers (e.g., tide gauge stations, marine protected areas, access points, eelgrass beds, etc.) over a backdrop of aerial photographs, political boundaries, natural resources, human uses, bathymetry, or other data. Users can create and share maps and download the actual data for use in desktop Geographic Information System (GIS) software.

MORIS, with its associated database and map server, is a working implementation of an interface to the underlying data. MORIS gives Massachusetts a solid first step in creating a data network. For example, much of the static geospatial data already exists in a normalized database that is can be searched and queried. As the data network comes online, the Arc SDE database and the GeoServer application are well placed to handle the static geospatial data transport tasking that the network would require. 
Because Geoserver can publish data in a variety of open standards, including KML and WMS, the data can be consumed by many applications which demonstrates tremendous interoperability and allows the system to be a key component of the data network. We evaluated a number of different examples of MORIS data being integrated in external applications:

- Using the GoogleMaps API to access MORIS data via the OGC WMS service. This integration makes a call to the MassGIS WMS server for an overlay of towns colored by particular data. The request includes information instructing the MassGIS WMS to color the towns based on particular data values. This procedure uses the GeoServer's styled layer descriptor or SLD.

- Using a Javascript API ( OpenLayers) and GoogleMaps

We did not evaluate the ability of MORIS to publish its data via KML which is a Geoserver option but also see that as a valuable connection option.

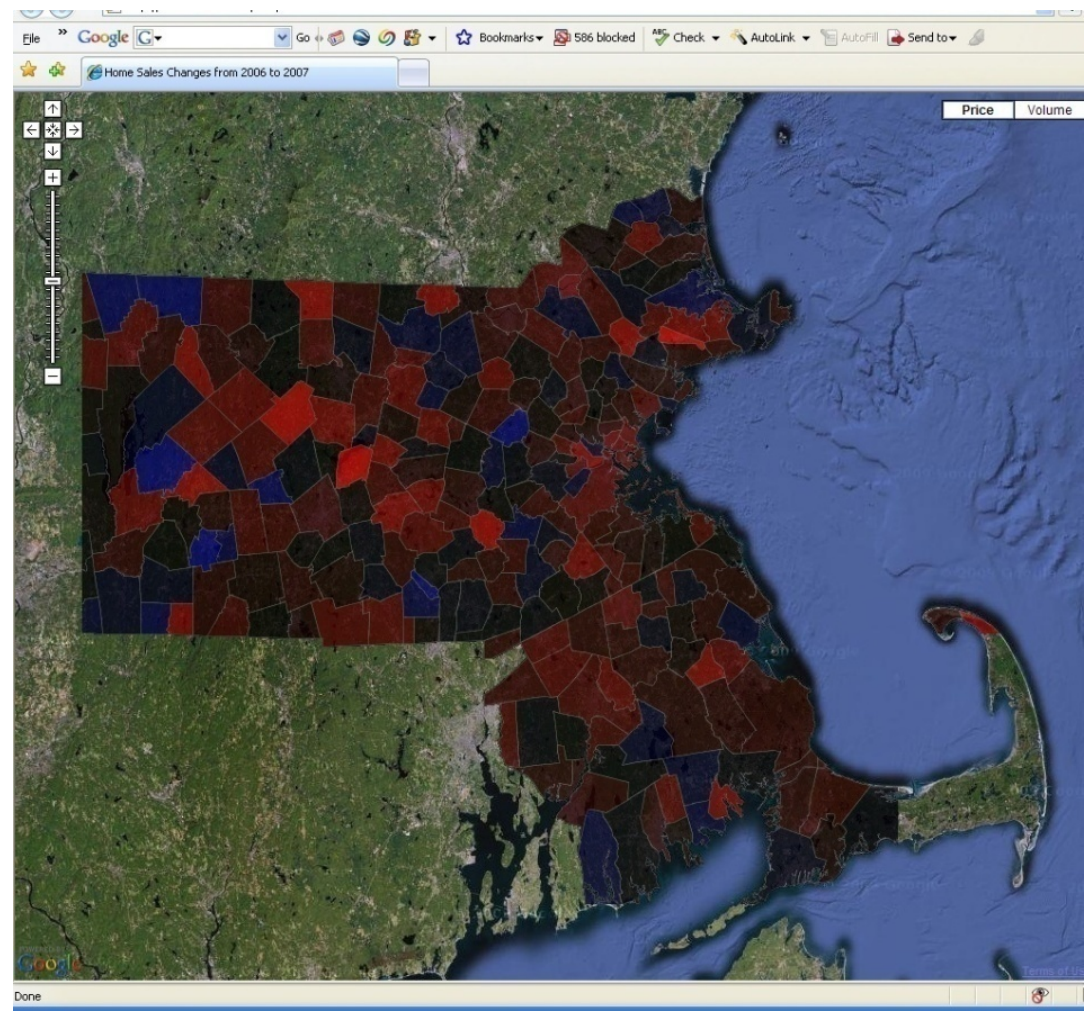

External Google Maps demonstrating integration using MassGIS WMS service. 


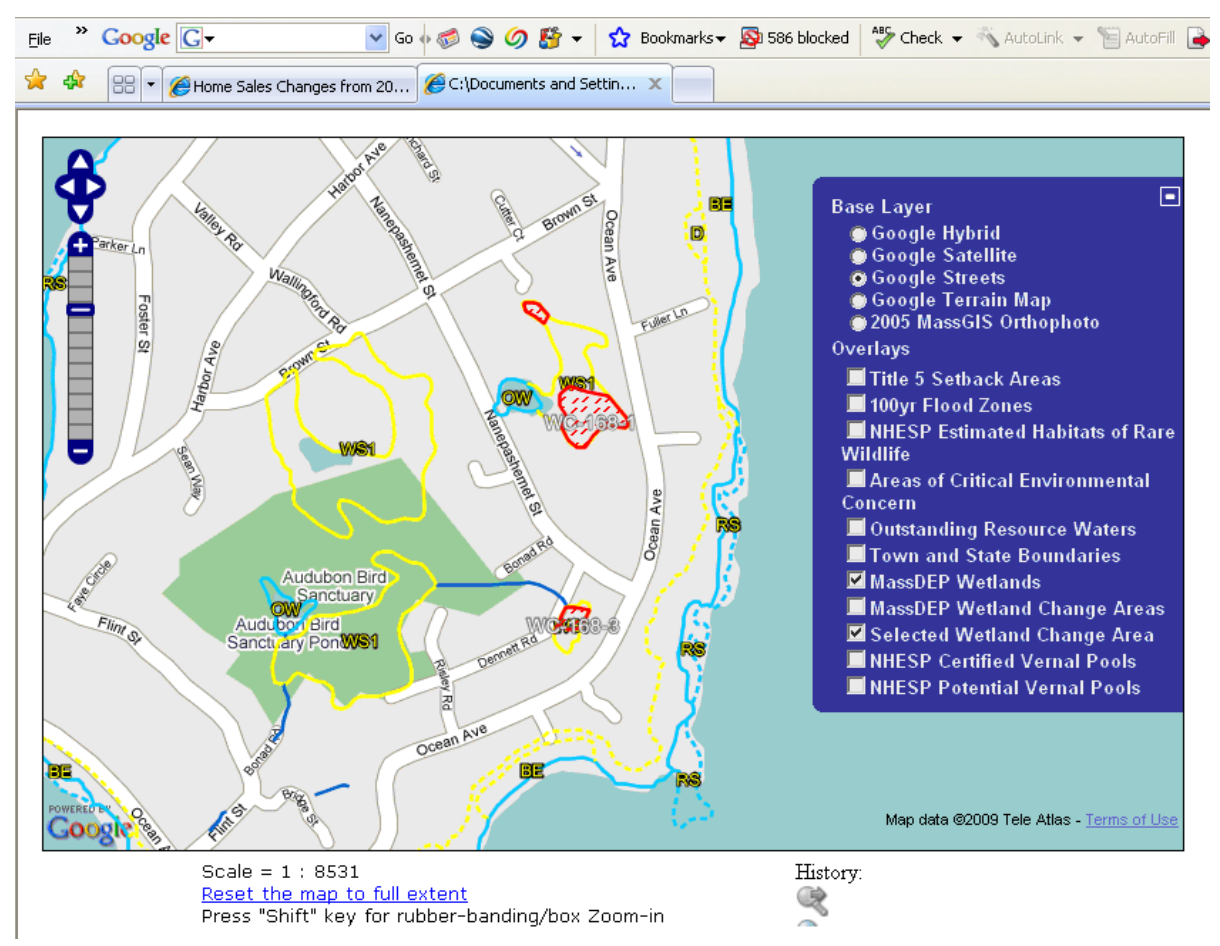

MORIS data being access through the OpenLayers interface

\section{Discussion of MORIS}

The pro's of the MORIS online tool are (1) on-line access to a large volume of data, and (2) the metadata is easily accessible (layer info tab) and well documented, and most importantly, the data is directly accessible via a suite of open standard formats.

However, the client application/tool (MORIS) that accesses the data would need some improvement for a broader audience, the tool responds very slowly and some functions are not optimum for viewing data. The main issue with using this tool is the performance, we would need further investigation as to whether this is the tool, the speed of the Geoserver services, or a combination of both. It is clear that the new generation of on-line map users are used to highly responsive applications, such as Google Earth, and the user expectations for on-line speed and performance are very high.

In terms of rendering, the tool also needs more flexibility and user options. For example, if a user wanted to view multiple State Regulatory boundaries, there does not seem to be an option to color code based on attributes, a feature inherent to the Geoserver Map Server, but not exposed through MORIS.

Another example that was encountered was the inability to visualize attributes such as lobster harvesting amounts symbolized based on a chosen attribute. 


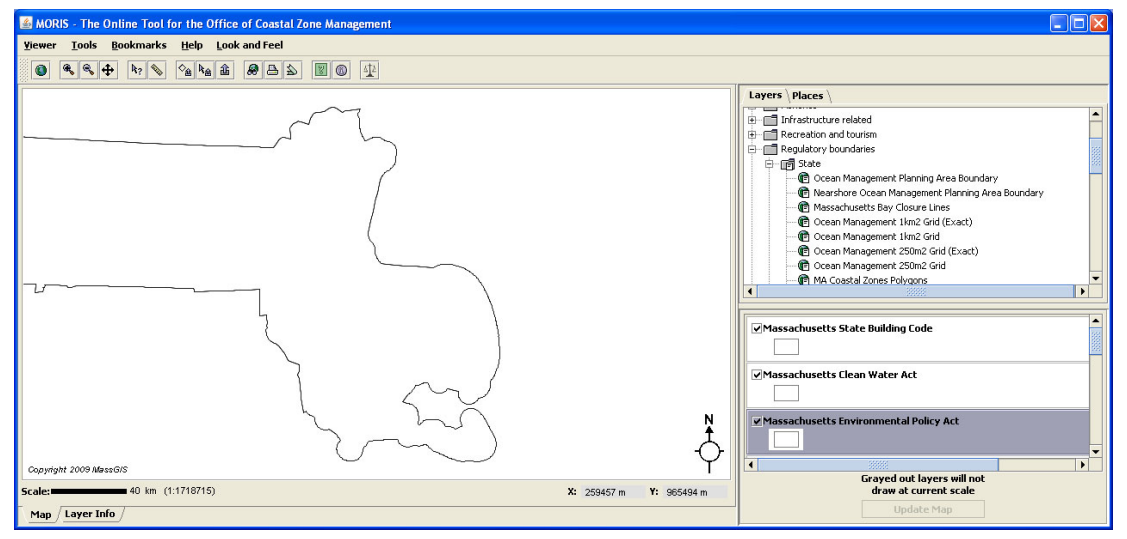

State regulatory boundaries

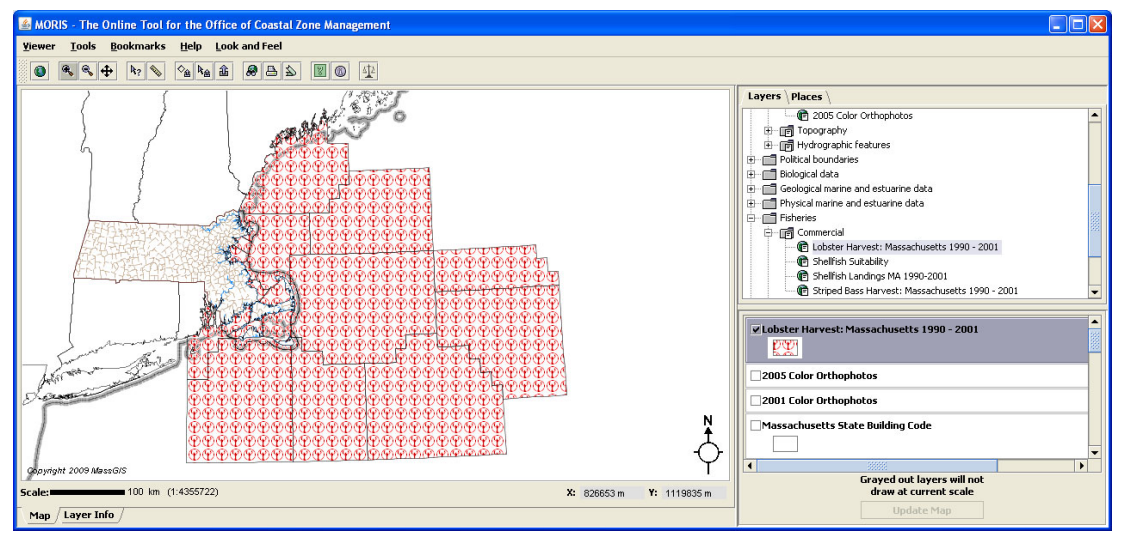

Lobster harvest information

\section{ArcGIS Server}

We endorse the use of Opensource tools such as Geoserver, but it is also useful to look at other options such as commercial tools like ESRI ArcGIS Server.

The ESRI solution includes data management capabilities providing services for data extraction, replication, and synchronization, as well as a framework and tools for managing large spatial datasets in an RDBMS such as IBM DB2, IBM Informix, Oracle, Microsoft Access, Microsoft SQL Server, and PostgreSQL. Further, programming the Common Data Source API allows for the development of unlimited custom data access objects. Additionally, the solution supports several web services including proprietary Map, Image, Globe, locator and geoprocessing services, as well as supporting standards such as KML, WMS, WCS, WFS and WFS-T. REST and SOAP protocols are included with the server solution, as well, allowing for access to the core functionality without the requirement to furnish ESRI client side products (such as ArcGIS Desktop).

The ArcGIS server solution framework also features an integrated IDE (Application Developer Framework -ADF) for developing in various APIs. The ADF is available for developers using .NET, Java, and Flex. 


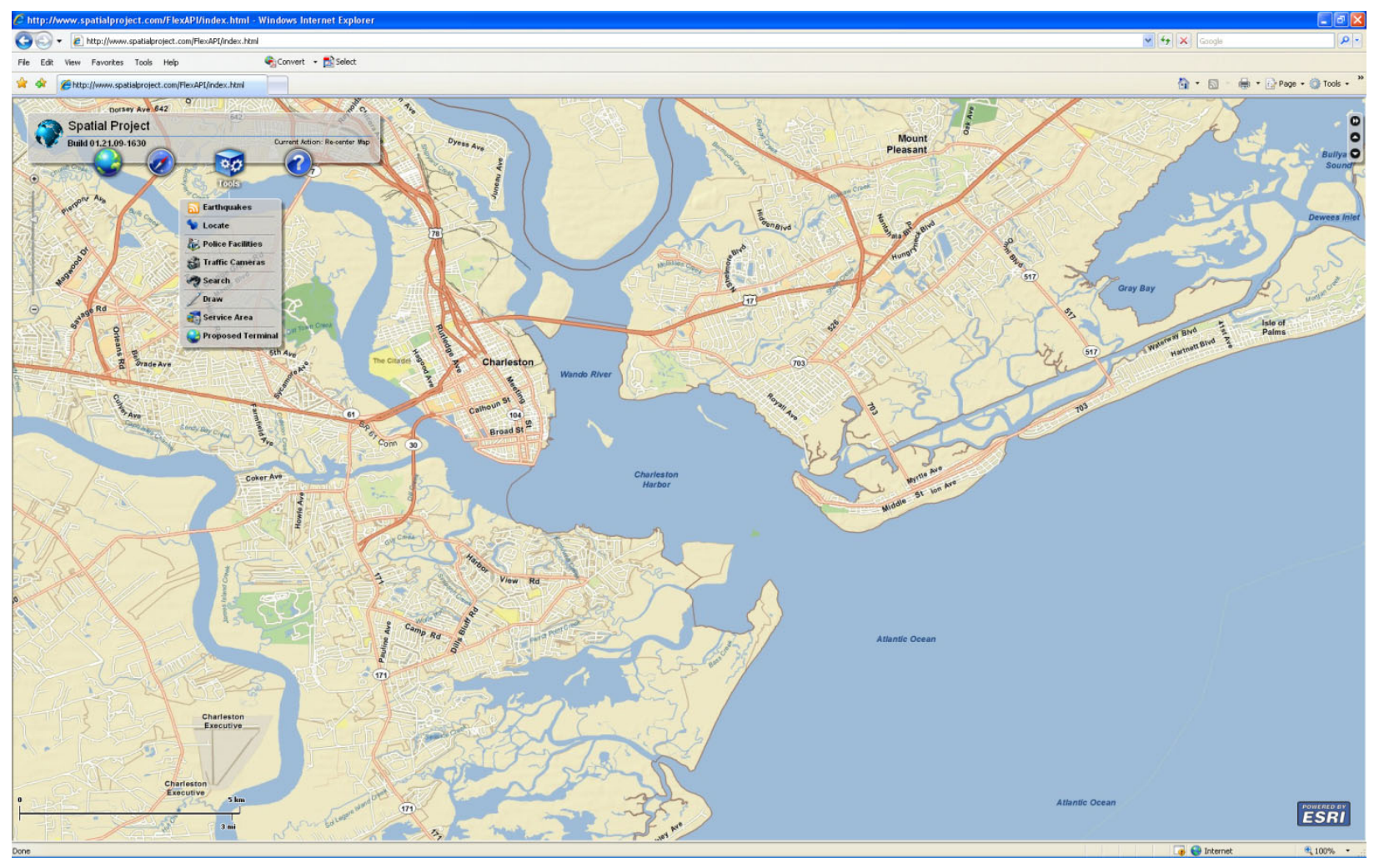

Example of an ArcGIS Server application built with Flex (USCG)

The solution framework supports capabilities for image data management and server side integrated caching and auto-tiling allowing for enhanced performance of client applications. Additional performance features are available through the customization of the rendering of the output. Applications can customize rendering at the web-tier graphics level (server side) or client-tier graphics (browser based) dependant on the type of data and the volume being rendered.

The framework supports server-based analysis and geoprocessing, including vector, raster, 3D, and network analytics, as well as models, scripts, and tools. The IDE provides various levels of access to the geoprocessing services, allowing for custom development and extension of existing services and tasks. Spatial analysis is built into the server framework including suitability analysis, cut-fill, line-of-sight, and terrain modeling.

The solution framework also features integrated web editing functionality, allowing spatial editing tasks for applications, such as adding, modifying and deleting map features like points, lines and polygons. As a result, applications can be stood up that allow various user groups differing levels of functionality and management capability from remote access points (the web). This allows fully integrated data management for an unlimited number of users without the need to further purchase additional licenses.

Although MassGIS uses SDE to store data, it may be possible to use ArcGIS Server in tandem with GeoServer. We see the ability to quickly build a variety of web clients as a significant advantage of the ESRI solution. We also see ESRI's new tiling and caching scheme as a significant performance improvement over previous versions. 


\section{Regional Point Observation Data}

The data from in-situ observations is the second data category. We classify this as data from stationary buoys and sensors, as well as drifting buoys and gliders. Although, there are many regional observations, our recommendation for this data category is that we leverage the national efforts and rapidly maturing standards for sharing in-situ data. As part of the IOOS Data Integration Framework (DIF), the use of the Sensor Observation Service (SOS) has been endorsed. SOS is a XML-based standard for sharing in-situ data. All of the Regional Associations are being advised to provide their in-situ data using SOS, and many of the regional observations are centralized at the National Data Buoy Center (NDBC) that now also provide national data using SOS. The current challenge with SOS is the lack of client software that consumes and digests SOS and makes it usable in applications such as Excel and Matlab. Middleware such as ERDDAP (Environmental Research Division's Data Access Program ) can consume SOS and provide the data in formats such as XLS and KML that are easily consumed by many clients. The data network must not only be able to recognize and consume SOS, but also ensure that a wide variety of users can understand and use the SOS data streams.

Providing a bridge to SOS does not solve the data management requirements for all in-situ observations. In order to best meet the needs of the diverse community of researchers, managers and operational users, the network should be able to interface with a number of existing and emerging protocols. There are a number of other observations that need to be addressed and interfaced with, such as real-time water data from the USGS National Water Information System (NWIS) and water quality data from EPA's Storet system. An initiative is under way, the Water Quality Data Exchange,that will allow web service access to USGS and EPA data. The data network must have the ability to connect to the Water Quality Exchange (WQX) format or the evolving WaterML XML-based standard.

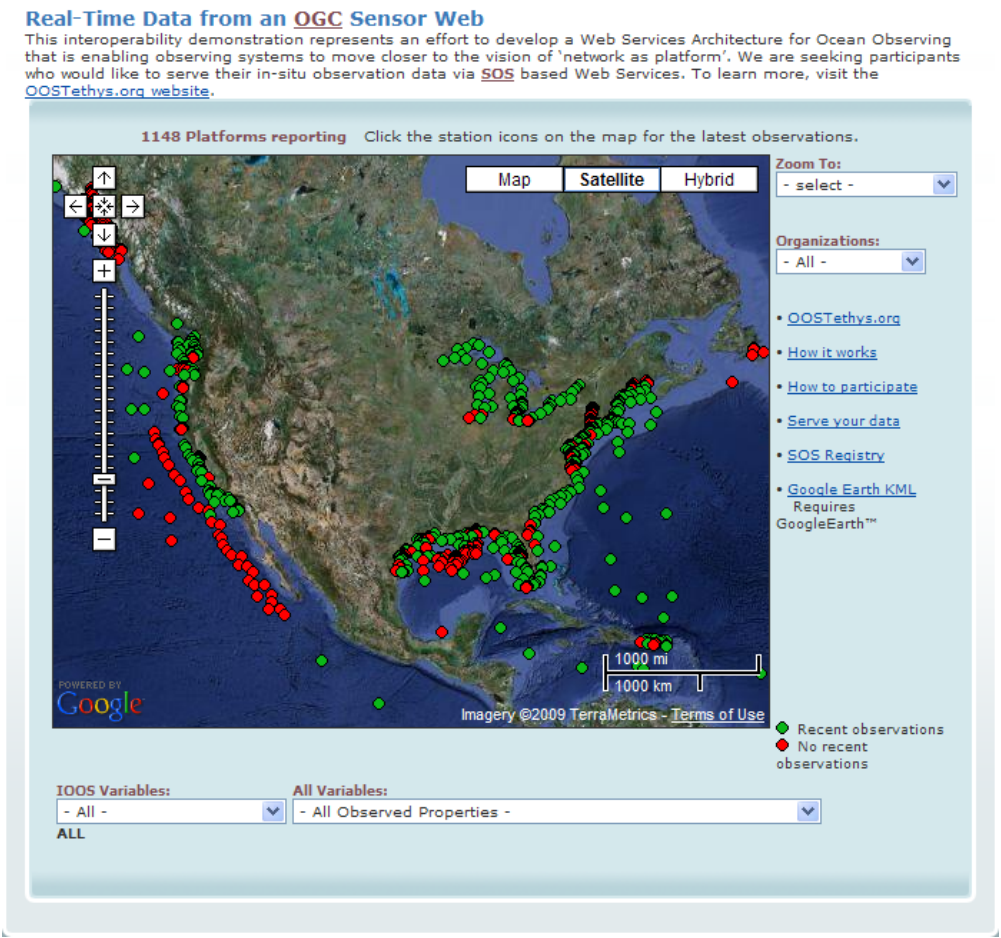

Openloos.org displaying in-situ observations using KML and SOS 
In conclusion the data network must have the ability to integrate and archive real-time in-situ point time series data. Thanks to a rapidly advancing federal network of Data Assembly Centers (DAC) such as NDBC, the ability for the network to connect to these centers as opposed to individual regional distribution centers will be most efficient.

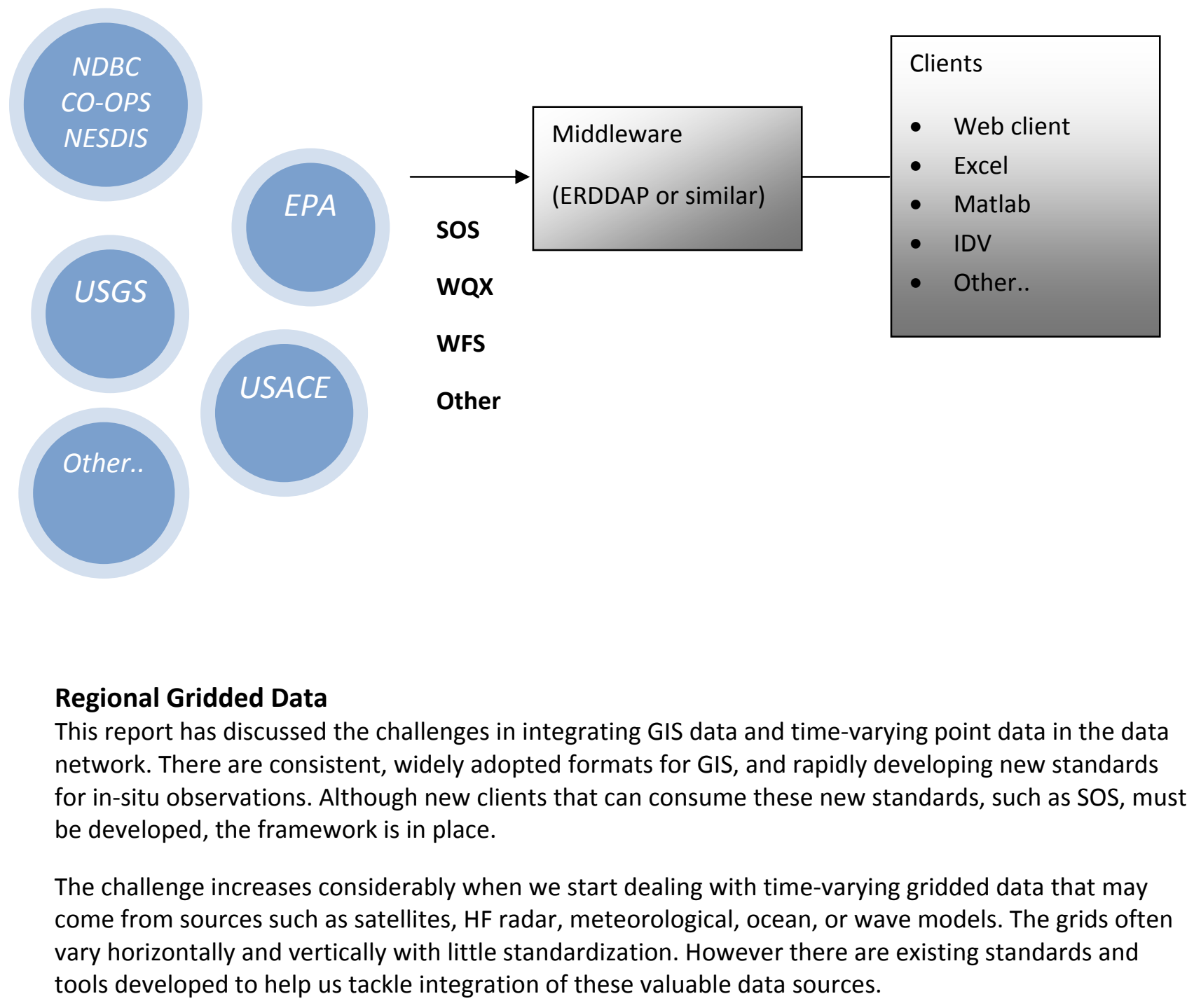




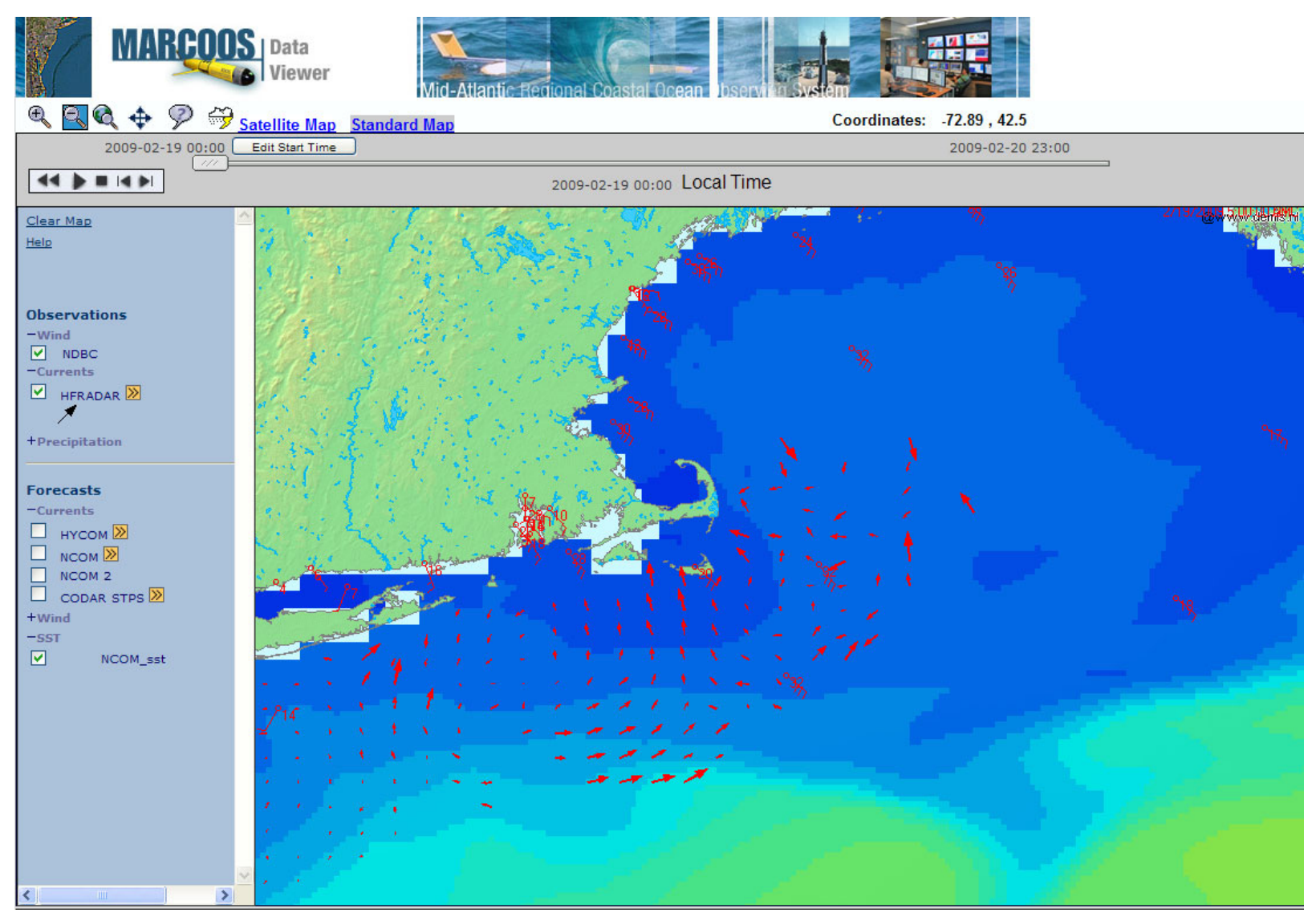

Gridded Sea Surface Temperature and HF Radar surface currents

Existing models which focus on the Massachusetts coastal ocean include the UMAINE GOM (POM) model, the UMASSB MWRA (ECOM-SI) model, the UMASSD NECOFS (FVCOM) model and the WHOI GOMTOX (ROMS) model. All of these groups have run hindcast simulations (retrospective forecasting using observed information; usually applied for model validation) which are a valuable resource for management. The technical challenges involved in analysis and application involve both the cyber infrastructure required to store and transmit the large (Terabyte) data sets, as well as challenges related to the peculiarities of each model, such as the data format and model grid. Management planning applications require mainly statistical information about the modeled environment, not the hour-byhour hindcast conditions. This can dramatically reduce the infrastructure challenge for the primary user. The data providers (model groups) must do the analysis of their local data, sharing the statistical results rather than just the raw hindcast data. The statistical data are small enough that when properly formatted to comply with existing standards they can be transferred using existing web-based technology such as THREDDS Data Server developed by UCAR/UNIDATA.

Using standards such as NetCDF, OPeNDAP, Thredds, WCS, and WMS will guarantee that the data network is interoperable with the OOI and IOOS initiatives. The remaining technical challenges to scientific model based management are really mathematical and statistical issues about uniform, comparable analysis methods which require a detailed understanding of ocean physics and each model. These issues must be addressed in cooperation with the scientists who developed and run the models.

\section{- 29 - INTERIM DRAFT}


This document describes four of the existing ocean models that focus on the Massachusetts coastal zone and outlines the technical gaps between the data formats available in the scientific model community and the needs of the management planning community. The conclusion outlines the steps to fill these gaps.

\section{Description of regional models}

There are four scientific numerical ocean models which have been used to do significant hindcast studies in the region. These models include various numerical and physical schemes to estimate water velocity and water properties in the region. Each model uses a different resolution and a different grid structure to resolve the physics of the ocean. Each model takes advantage of certain strengths for a particular application. Judging which model provides the best answer for a particular problem requires a great deal of scientific understanding. One of the goals for the data network development should be some consensus on how to compare the parameters estimated by the models. The cursory examination of the modeling efforts below is only to highlight some of the most obvious differences between the available data.

MWRA : UMASSB - ECOM-SI

The Massachusetts Water Resources Authority (MWRA) employed UMASS Boston to hindcast water quality in Boston Harbor and Massachusetts Bay. The UMASS Boston team used the ECOM-SI model with a curvilinear grid (a grid bounded by curved lines) covering the Bay from the New Hampshire Massachusetts state line to the Eastern shore of Cape Cod. The curvilinear grid is 68 cells on each side with the highest resolution (600 meters) in Boston harbor. The hydrodynamic model uses 12 horizontal layers to capture the vertical structure of the water column. The water quality study included both a hydrodynamic model as well as a biological model to study eutrophication, the build up of the concentration of plant nutrients. The model boundary conditions are based on available data including meteorological and oceanographic observations. Several years of model data are available in NETCDF files, which are readily accessible by existing web service technology. 


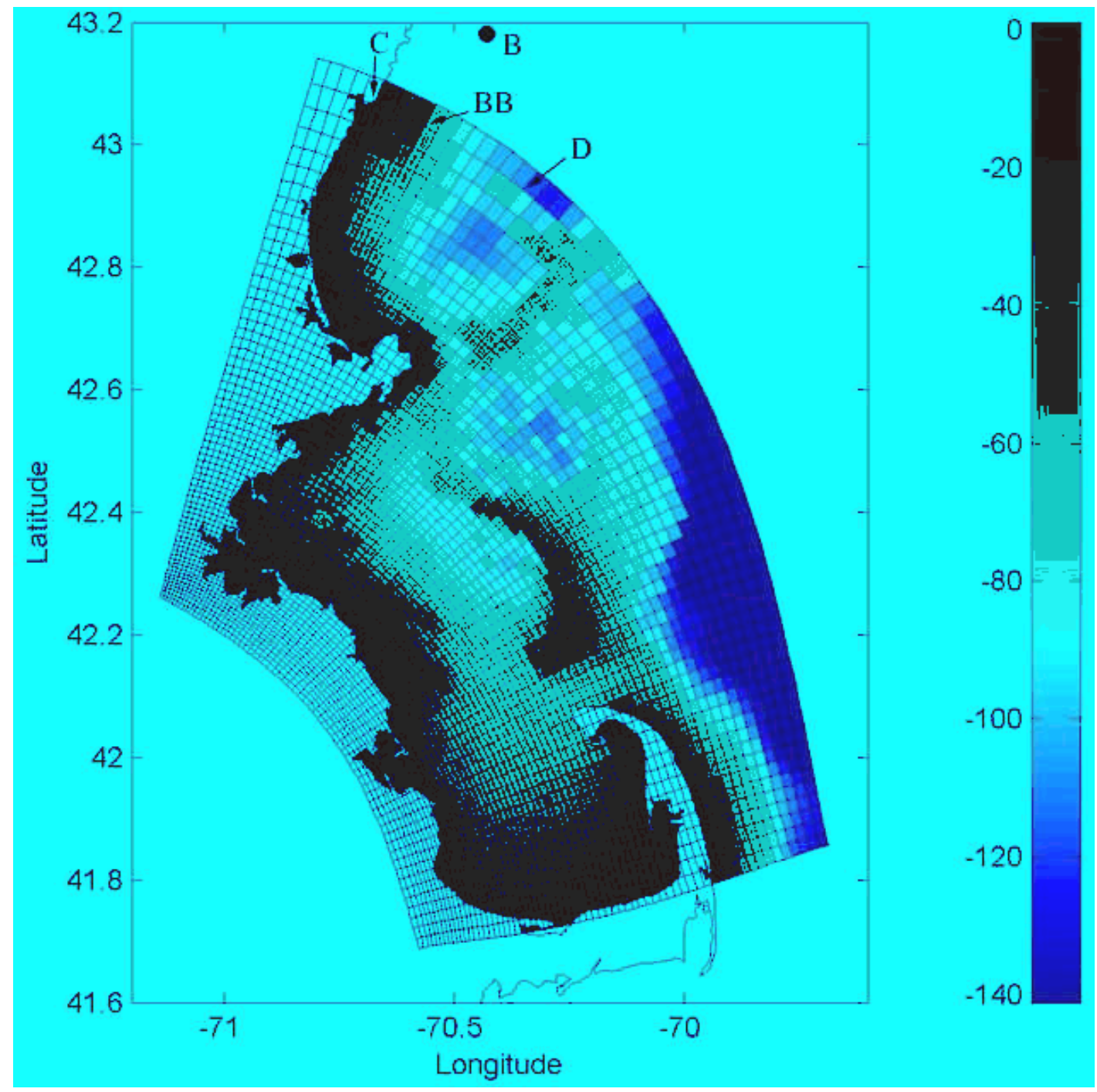

ECOM-SI model grid and depth in meters.

\section{NECOFS : SMAST - FVCOM}

Through physical process studies and fisheries management research the Marine Ecosystem Dynamics Modeling (MEDM) group at UMASSD/SMAST has developed and applied FVCOM to hindcast the Gulf of Maine and Georges Bank including the Massachusetts coastal region. The model domain extends from New Jersey shelf to the Scotian shelf. There are several generations of the model domain with successively higher resolution in the coastal region. The number of cells in these grids range from tens to hundreds of thousands. Due to the unstructured triangular grid the resolution can be as high as a few meters in coastal regions. FVCOM typically uses 30-40 horizontal layers to resolve the vertical variation of currents and water properties. The FVCOM GoM hindcast studies have relied on a meteorological model hindcast to determine the surface forcing and available observations to force the open boundary of the model. While the MEDM group continues to use the model as a research tool, they have also developed an operational forecast system. The data are stored in NETCDF files but there are still no implemented standards for unstructured model data in the earth science community so the format is

\section{- 31 - INTERIM DRAFT}


unique to the FVCOM model. The data can be accessed using existing web technology but tools to work with the data are very limited.
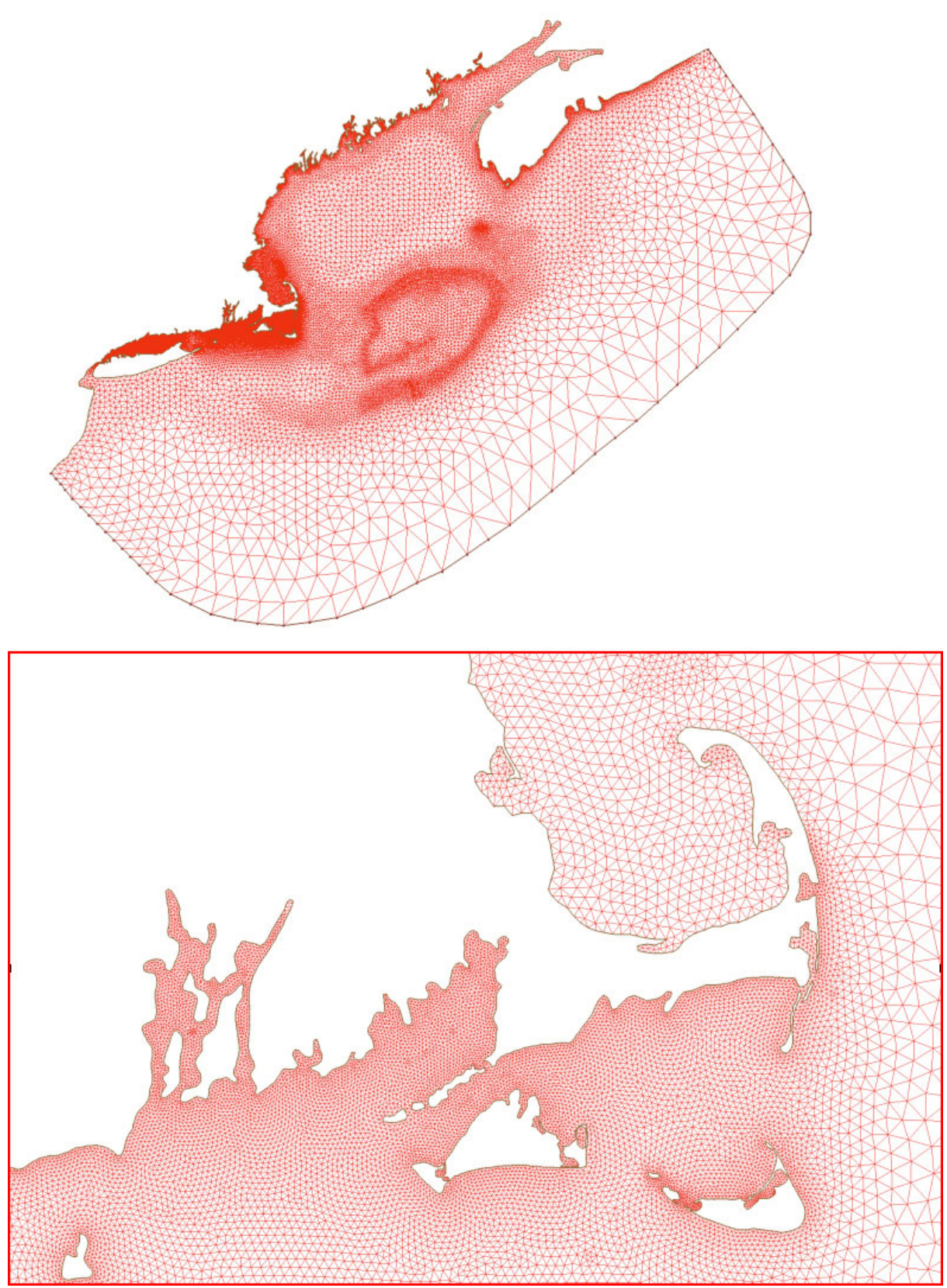

FVCOM 2nd Generation model grid

UMAINE GoMOOS : UMAINE - POM

Researchers at the University of Maine applied the POM model to the Gulf of Maine region to study the dynamics of the biological and physical environment. The curvilinear domain covers the region from the Scotian Shelf to the Rhode Island - Massachusetts border. The model has $103 \times 151$ horizontal grid points with the highest resolution $(\sim 3 \mathrm{~km})$ on the Maine coast. There are 19 horizontal layers which resolve the vertical variation of water properties and currents. The model is used in the GoMOOS

\section{- 32 - INTERIM DRAFT}


forecast where it is run daily with meteorological forcing from a NOAA mesoscale model, and Atlantic Real Time Ocean Forecast System data on the open boundary. The POM model results for the GoM region are available on the GoMOOS site and through the UMAINE web site as NETCDF files.

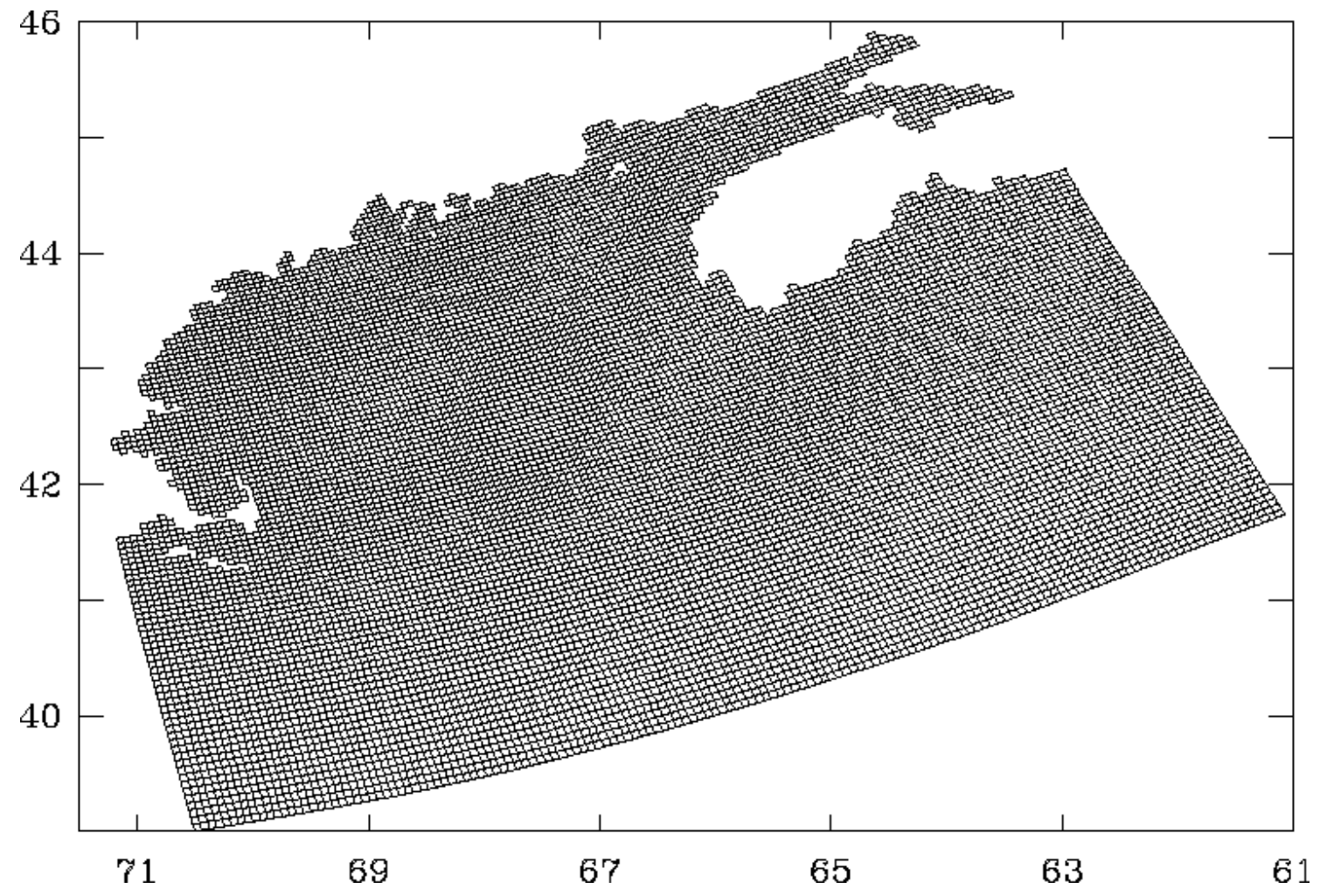

\section{GoMTOX: WHOI - ROMS}

The WHOI GOMTOX project in collaboration with other research groups uses a nested ROMS model to study hydrodynamics and red tide in the Gulf of Maine. The rectangular grid of the high resolution GOM nest is made up of $1 \mathrm{~km}$ cells covering the region from the Scotian shelf to the Massachusetts - Rhode Island state line. The model uses 36 layers to resolve the vertical variation of currents and water properties. The surface forcing for the ROMS model comes from a mesoscale meteorological model while the nested approach provides the open boundary conditions. The ROMS model has advanced data assimilation methods which help improve the accuracy of the model relative to available data in the region. The model output is in NETCDF files which can be hosted using web based technology. 


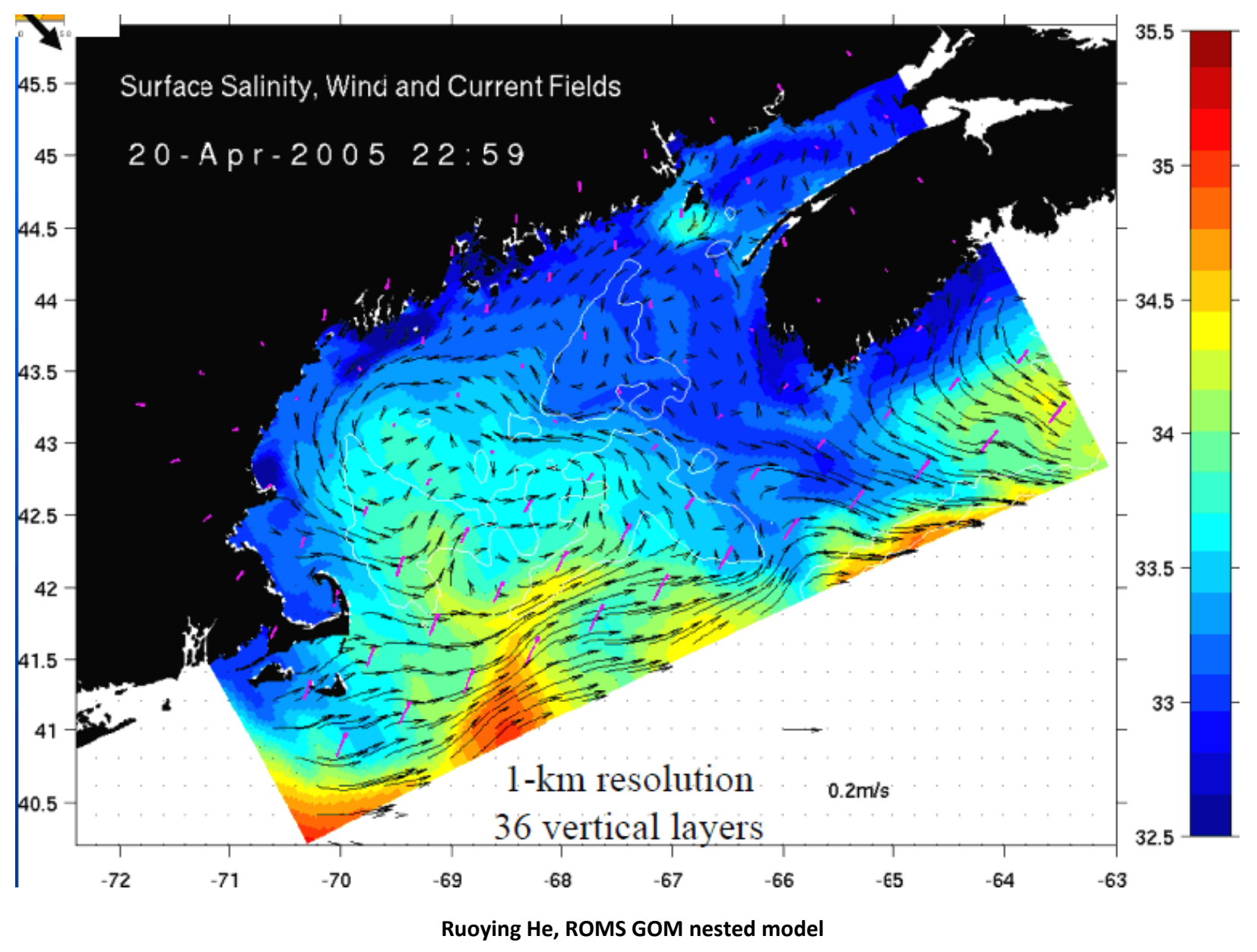

\section{Challenges for Models in Data Network}

The summary of the model hindcast studies describes some basic features of the scientific research efforts. Each model has different coverage, different gridding, different forcing and different physics. The technical challenges in using the available model data to inform coastal zone management require a scientific discussion of how to use and compare the data. The user must carefully define the parameters of interest and work with the scientific model groups to determine how to estimate these parameters. For instance, the bottom stress and bottom velocity from different models are calculated using different physical parameterizations and different assumptions about the bottom roughness. These differences may appear subtle but the estimated bottom stress may be different by an order of magnitude. This is not an issue of model accuracy and precision but rather the definition of the parameter. There is a further question of validation of the model results using real data. This scientific challenge may require significant political negotiation among the scientific groups which are competing for funding. To apply model results in coastal zone management, the scientific modeling community must be engaged and work together with the state management team. The definition of the parameters is really a scientific question but also a critical aspect of the technical challenge to using model data in the MOP data network. 
As important as the definition of the parameter, the location of the data in the model grid also requires significant insight into the physics and numerics of the model. Ocean models simplify the vast ocean which varies continuously on scales from millimeters to thousands of kilometers. The model describes some simplified parameterization of the physical reality throwing out certain aspects as less important by assumption. The equations which describe this simplified world are then discretized so that a computer can solve for the temperature or velocity at points in space and time. Each of the model hindcast studies uses different mathematical tricks to improve the estimate or find the solution faster. As a result the models do not estimate the ocean parameters in the same way or at the same grid points. The grid structure is a key issue which makes it difficult to analyze and compare the results. For example it is not possible to directly estimate the maximum bottom velocity in Boston harbor by combining results from both the FVCOM model and the ROMS model because they do not estimate it at the same locations. While it is possible to qualitatively compare maps showing the model results any further analysis will require interpolation to a common grid where values can be compared point by point. On the other hand the native model grid data is required for any detailed analysis of the model results. For model validation and use in the scientific community raw model time series are essential.

\section{Using existing cyber infrastructure}

By limiting the scope of the initial requirements for the data network to spatial statistics rather than analyzing the variability over time, the technological challenge is greatly reduced. Each of the hindcast studies has seasonal and inter-annual time series. These data files may be hundreds or thousands of gigabytes in size. The data network will need the cooperation of the model groups involved to process the data and solve the scientific challenges described above, but also for the technological requirement that the raw data is too large to work with through the internet. While server side processing technology is under development, tools available in the near future will not be sufficient to process the model data time series as a web service for management purposes. The statistical maps that are generated by the model groups can easily be shared on any web server. The NETCDF JAVA libraries provide the tools to access regular gridded model data on any http server in a flexible and user friendly manner. To catalog a larger group of results in a more flexible way, one or more THREDDS servers could provide a uniform point of access to all of the model results. The THREDDS data server uses the same NETCDF JAVA library to access local and remote files providing an interface through a web browser and in other client applications.

The GoMODP has already started an interoperability study for model data lead by Rich Signell at the USGS in Woods Hole. The USGS THREDDS server hosts example model data from all four of the significant hindcast model studies cited here. This software developed at UNIDATA removes most of the technological impediments to sharing ocean model data. Once the data is hosted in this way it can be accessed through a number of tools for visualization and analysis. The tools have been developed based on conventions for rectangular and curvilinear gridded data models. If the data network requests data in the native grid of the model then the data will still be accessible through THREDDS but the software available to work with the data will vary from model to model depending on what conventions it follows. The FVCOM model is the most difficult case because it uses an unstructured grid setting it apart from the other models. While conventions are under development for unstructured model data they have not been adopted in any meaningful way. None of the software application tools support an unstructured convention yet. 


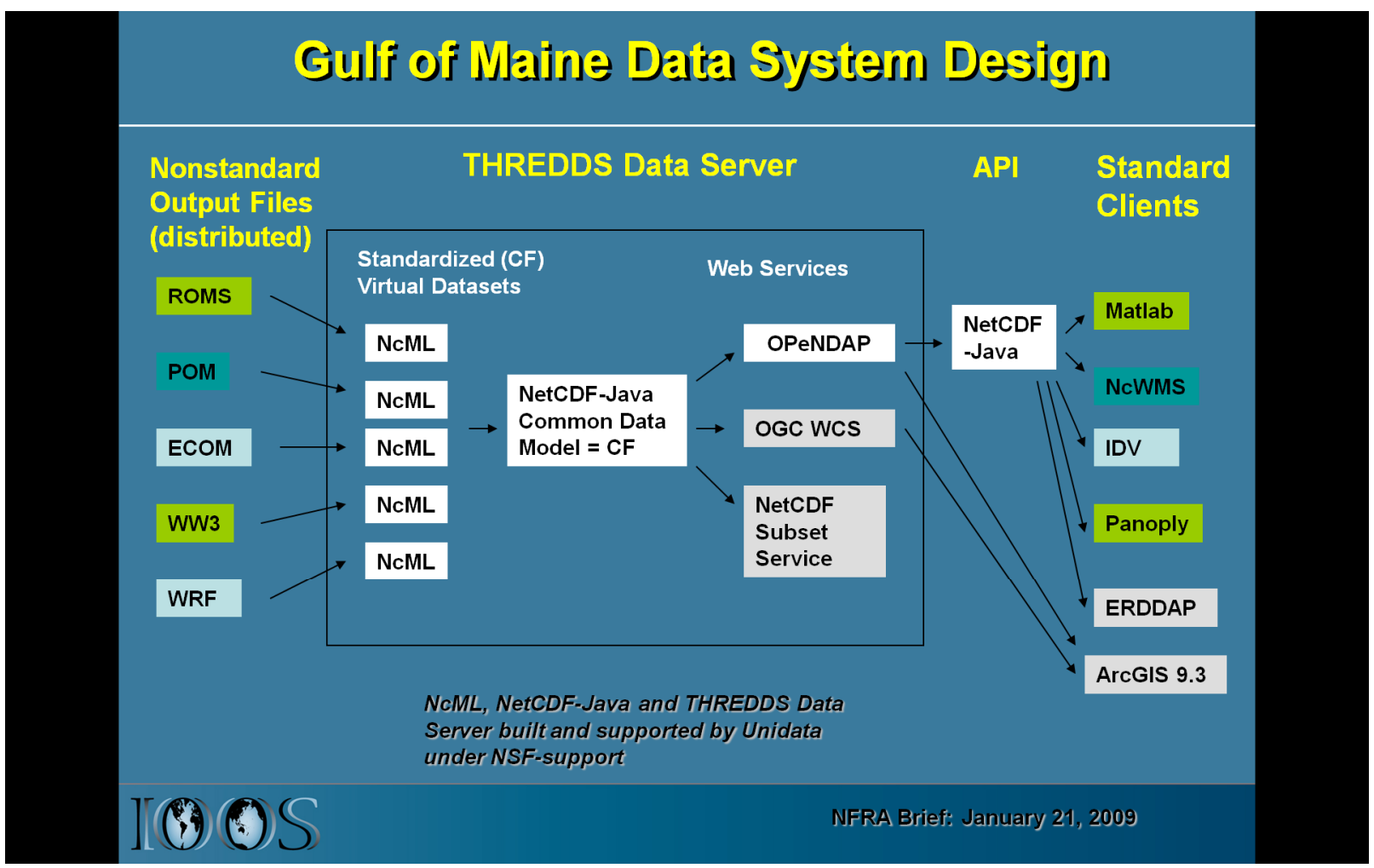

GOMODP Architecture for sharing model data (Rich Signell)

\section{Future Development}

The data network should support both the native model grid data formats for scientific purposes as well as a common coastal zone regular grid data format for management purposes. To fully exploit the use of data from the native grids, we recommend continuing standardization and new tool development in coordination with the GOMODP. A standard data model has been described for this purpose but has yet to be finalized as a formal Climate and Forecast (CF) convention. A tangible milestone that the MOP effort may consider supporting is the development of a working visualization package based on the proposed convention. Having a working visualization tool would give momentum to the convention process and allow the data to be more readily usable by managers and researchers alike. 


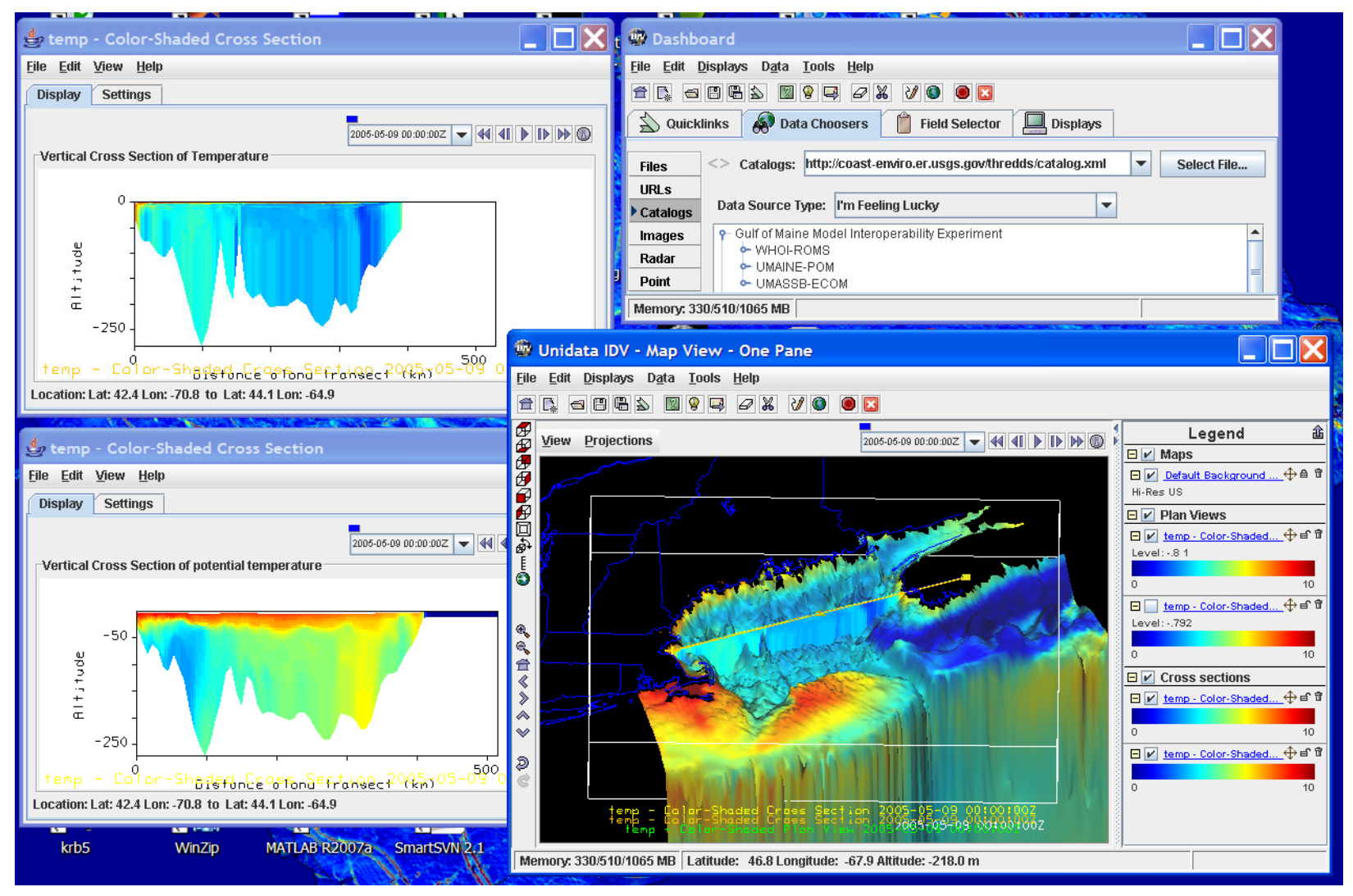

GoMODP, Rich Signell IDV demo

- 37 - INTERIM DRAFT 


\section{Data Network Design/Paradigm}

The design concepts that we have focused on for the data network are:

- Focus on interoperability and the use of existing science tools connected to commercial Web 2.0 concepts

- Leverage existing global, national, and regional efforts and use the general guidelines provided by the National IOOS DMAC/DIF concept of operations

- Provide tools and "glue" to integrate legacy systems as opposed to redesigning components

- Focus on meeting user's needs

\section{Focus on Interoperability and the use of existing science tools connected to commercial web 2.0 concepts}

Leveraging Web 2.0 technology will be most important in allowing data search and discovery which at present is a severe limitation. A number of data repositories and portals are in use by scientists and GIS specialists such as NASA's Global Master Change Directory (GCMD) and Geospatial One Stop (GOS), as well as many specialized metadata portals such as OBIS and MERMAID. A report by Stone Environmental discusses these catalogs and portals in more detail. The reality is that most users and indeed many scientists use traditional search engines such as Google. We see an important goal is to leverage the power of the Google search engine to allow a variety of end users to search and find ocean data that is geospatially referenced. A further consideration is including data that is informational but may not have specific geo-reference information, only a general location, e.g a report or video on Buzzard's Bay.

This challenge is not unique to Massachusetts. The concept of georeferencing disparate data such as reports, time series data, images and video data has been discussed for many years and the concept of the Geoweb is now a reality. The Flickr web site (www.flickr.com) is a widely used example of georeferencing data as simple as photographs.

This is just one example of the concept of a Geoweb (Hanke and Dangermond). Google has adapted their search technology, adding geographic references to its search functions and this allows users to perform geographic searches, essentially adding geographic intelligence to its traditional search capabilities. Google's GeoSearch, which is now a publicly available toolkit, allows web developers to build tools which add Google's geodata search to applications. This is enormously powerful and provides users the ability to connect to actual data through traditional Google searches. The search results can be accessed in tools such as Google Earth, or web sites that use Google Maps, Virtual Earth or any tool that supports KML and can access the Geosearch AJAX API. 


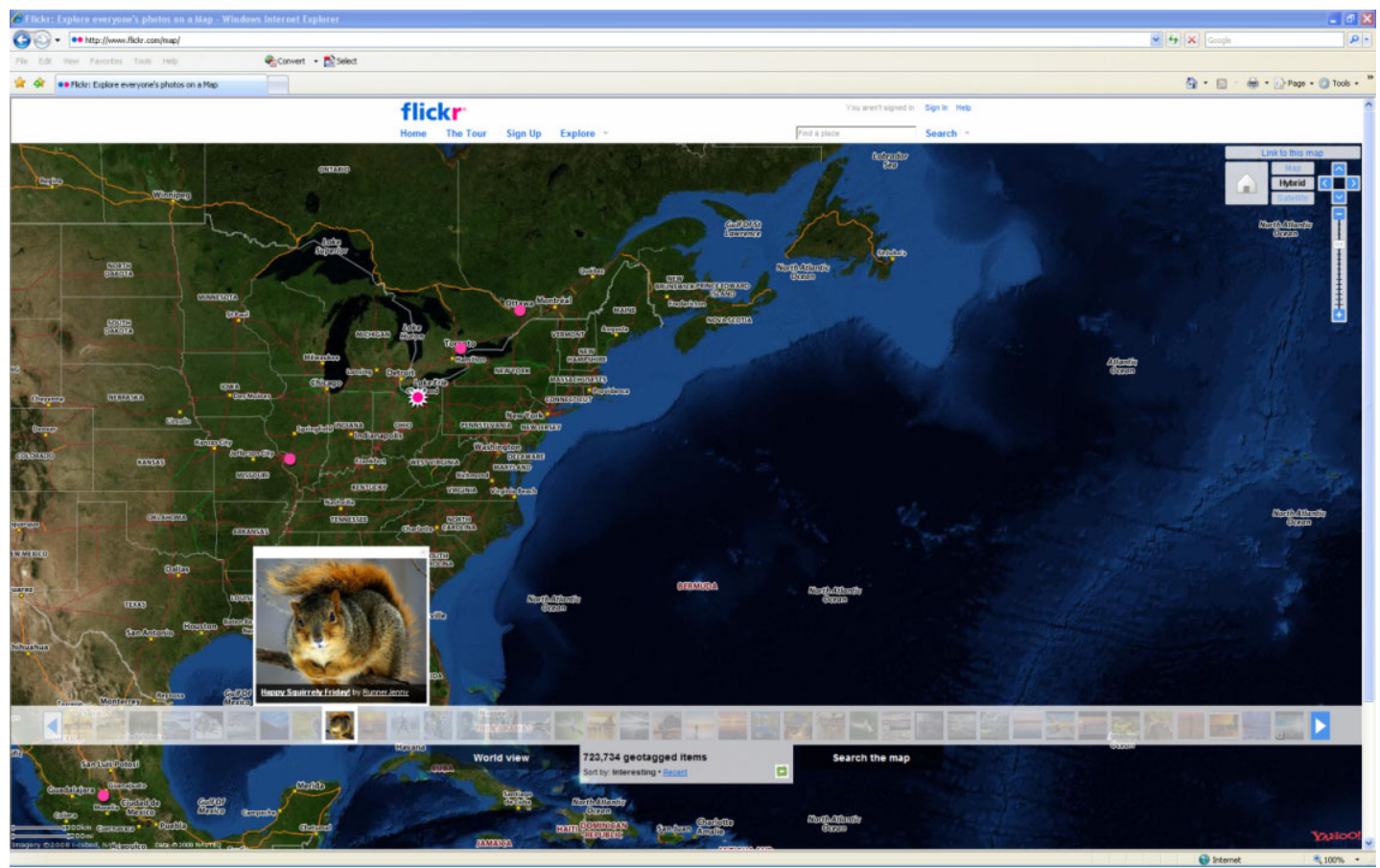

Example of Flickr web site that allows users to "geotag" and publish their photographs

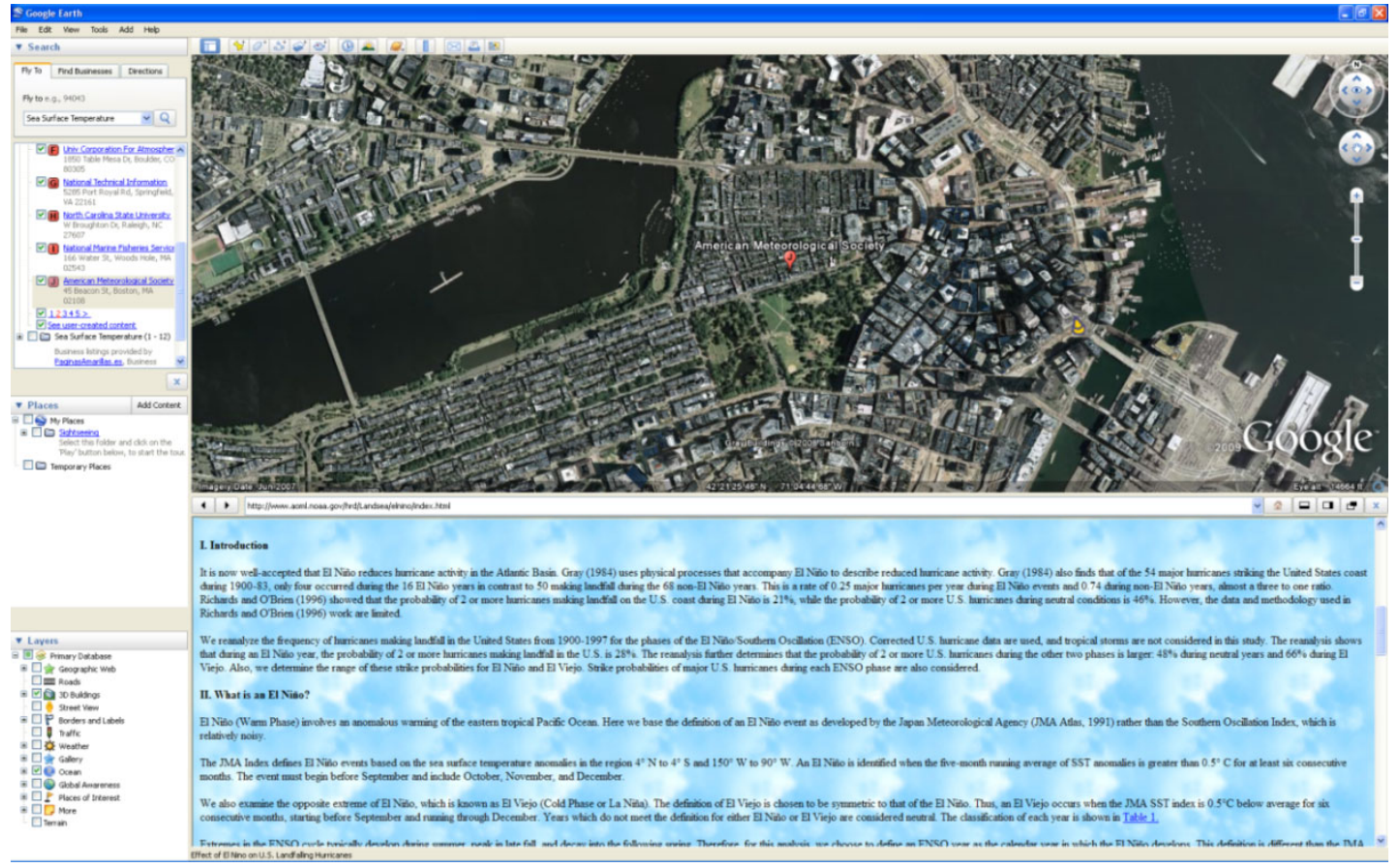

Example of searching for "sea surface temperature" in Google Earth 
The basic steps for publishing geo content with Google's search engine are as follows:

1. Create the KML or GeoRSS content. It is important to add attribute tags, which will appear in the Google Search results for the content. Metadata may also be added in the <Metadata> section.

2. Post the files on a public web server.

3. Create the Sitemap file and copy to the root directory of a website.

4. Submit the Sitemap to Google.

Having followed these steps, data providers can now make their data searchable by the Google search and locatable in Google Earth, Google Oceans, and Google Maps. In general, the KML should not contain all of the data but be a placemarker, or metadata, that points to the actual data. Although the Google geodata search may contain a web pointer, that redirects the user to other sites, it can do more than a traditional metadata portal. Using middleware/web services, the user should be able to discover the data through the lightweight KML and then connect directly to the data by simply clicking on the map. The map click would either reveal the data or direct the user to a set of web-based tools that will allow access to the data in different manners, such as images, time series graphs, map overlays, document viewing, or data download. These web-based tools are the "glue" described below.

\section{Leverage existing global, national, and regional efforts and use the general guidelines provided by the National IOOS DMAC/DIF concept of operations}

Systems projects that focus on the creation and implementation of standards appear elegant. All the data providers and data consumers know and use the same format. For systems that are new, this method is ideal. For a data network which is essentially a system of systems including an extensive array of legacy standards, protocols, and users, the use of middleware to connect users to data with minimal impact to the data provider is more effective, and quicker to implement. This methodology leverages enormous effort in different communities, ranging from ocean scientists to commercial organizations such as Microsoft and Google. This report does not recommend that new standards and data protocols are developed, but rather the adoption of a variety of technologies available from user and provider communities, including science, GIS, and modeling. Web 2.0 leverages the interactive nature of the Web and the concept of disparate users cooperatively delivering content for shared use. This is exactly the concept that is needed in the domain of ocean data to allow various user groups to connect to disparate data.

This report has discussed a number of initiatives that already embrace this philosophy, specifically the GOMODP which has endorsed a middleware example, the use of NCML to allow a wide variety of users to connect to a variety of legacy data. If this approach is tied to existing GIS data stored in MORIS for example, and if this data is readily searchable through the implementation of metadata and cataloging leveraging commercial search tools, the result will be significant access to data previously hidden from most users.

The data network should use, endorse and embrace open standards such as KML, WMS, WFS, SHP, NetCDF, GRIB, SOS and other open standards; this will allow seamless connection with national efforts. 


\section{Provide tools and "glue" to integrate legacy systems as opposed to redesigning systems}

We have focused on a top down approach based on the user's needs. The simple key to success for an ocean data network in Massachusetts is that a wide variety of users can quickly find and get the data they need in a format that they can use.

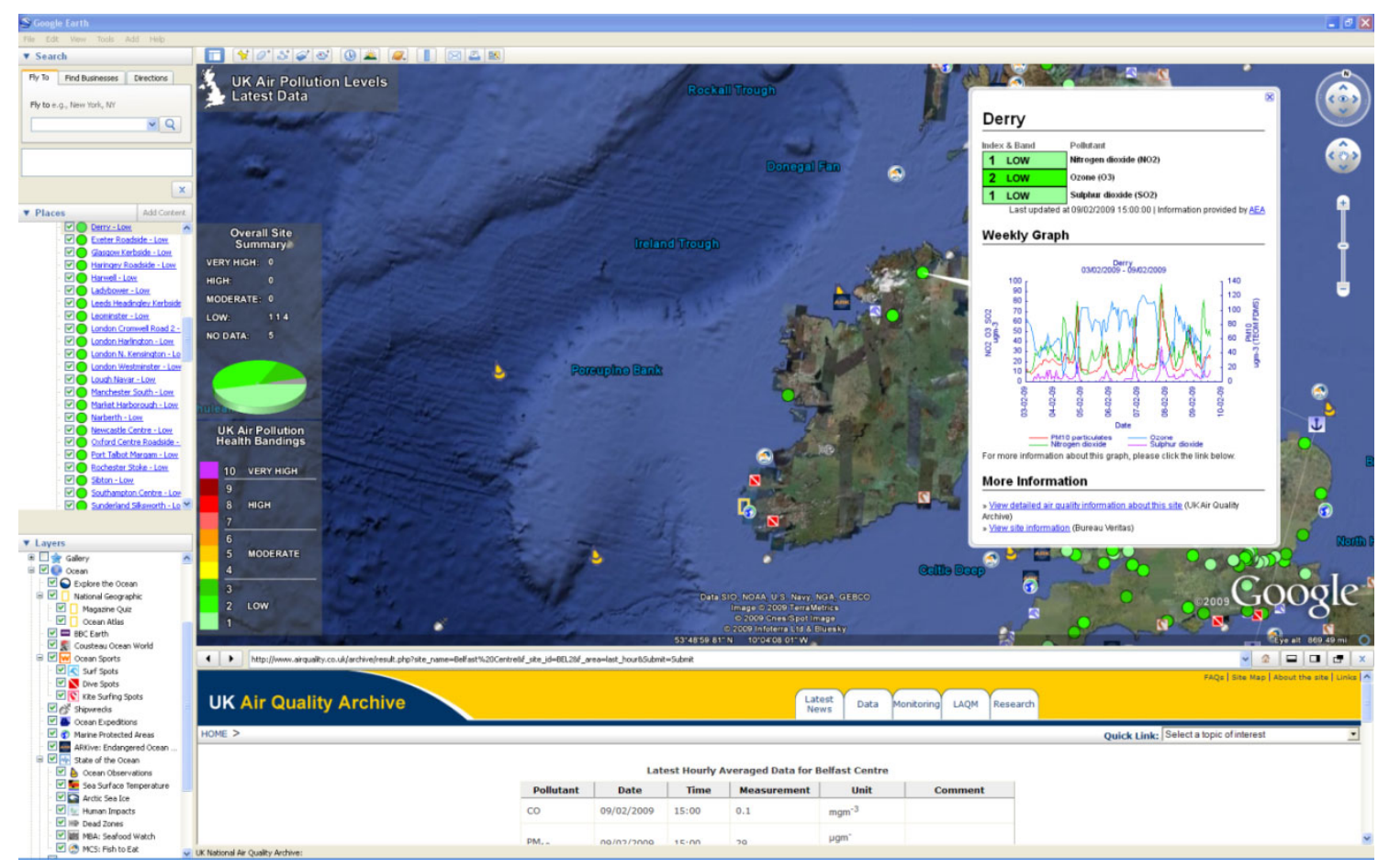

An example of Google searchable data network connected to actual data

The steps that we suggest to create this glue are:

1. Expose metadata and data sources via KML so it is geo-searchable. Where possible, automate the KML generation by creating tools to extract records from existing OPeNDAP inventories, THREDDS catalogs, and other metadata catalogs including GCMD.

2. Build a simple web tool to create KML geodata and expose users' data (reports, excel sheets, images, survey data, etc.) through a simple, standard method, and educate the data providers in its use.

3. Create an easy interface to access the data integrated into the search tools. Data "discovered" in a web search, Google Maps or Google Earth should have point and click access for standard image format (JPEG, TIFF, AVI, etc.) or document (DOC, PDF, XLS, PPT). For "discovered" data that is not typically consumed by standard applications (NetCDF, SOS, WQX, WaterML, WFS, DAP, WCS, etc.) the browser will connect to a web service, such as ERDDAP, that will allow the user to access the data as an image, a time series, raw data, Matlab format, ESRI format, or some other format used by scientists and GIS specialists.

\section{- 41 - INTERIM DRAFT}




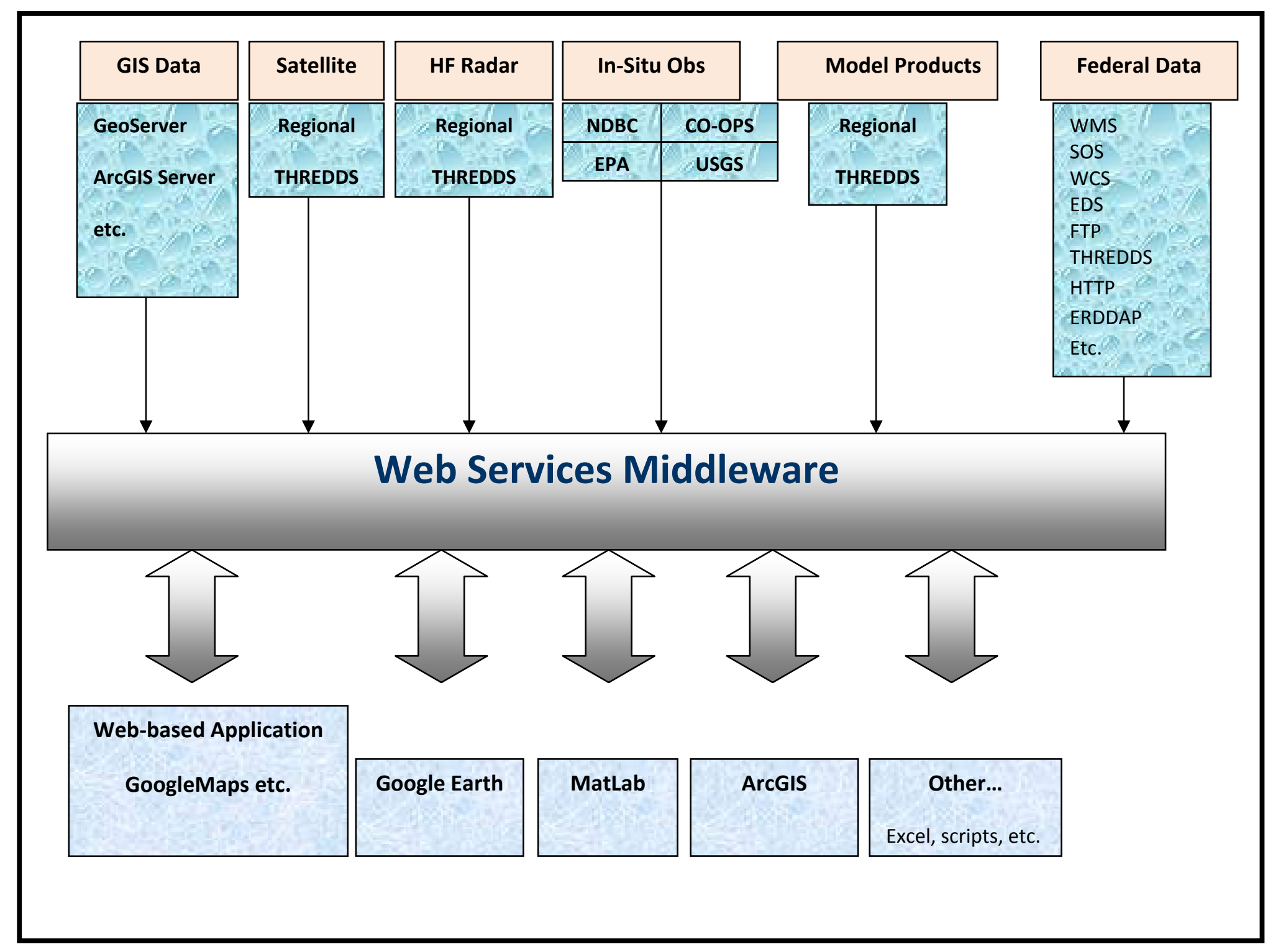

Using middleware to connect disparate data to disparate client applications 


\section{Focus on Meeting User Needs}

Many of the existing data infrastructure efforts are focused on the low level distribution of the data, web services standards, metadata standards, and transport protocols; we suggest exploring existing tools and interface layers that will allow users to get to existing data and minimize the changes to legacy systems. The next step is to define "use cases" for different user groups that efficiently allows users to perform screening level analysis using the available data. As an example, "show me all the regions that may be available for renewable energy projects" based on a set of rules applied to the data on the fly. The key to the success of the data network is in understanding the needs of the different user groups and connecting them to the data with appropriate tools.

\section{Network Design Conclusions and Recommendations}

1. Traditional static geospatial data for the State should continue to be served via MassGIS. They effectively manage the metadata and data distribution. Discussion needs to be arranged with MassGIS and the various regional data providers on the most efficient methods to get data into MORIS, perhaps an automated way to minimize overhead at MassGIS could be explored. We look forward to the next version of GeoServer 1.7.3 which supports metadata discovery via Google's GeoSearch.

2. The performance of the Geoserver services need to be improved, whether that requires a hardware upgrade or Geoserver software improvements would need to be determined.

3. Evaluate the pros and cons of using ArcGIS Server 9.3 in parallel with GeoServer.

4. Most users, even science users, do not use NASA's GCMD to find data. It is hard to use and strict metadata standards are required for the GCMD to catalog data. Similarly, Geospatial One Stop does not have significant traction with GIS users and is also difficult to use. We recommend focusing on the use of the Google search tools to find data, and as described above, expose existing catalogs to the search engine. This means that data must be exposed to the GoogleBot as described above. We recommend the development of tools to create and expose KML metadata automatically for existing servers (Geoserver, OPeNDAP, THREDDS, GCMD, GOS) and streamline the process to post data automatically.

5. Work with the science community and Google to define metadata requirements that can be embedded in the $\mathrm{KML}<$ metadata $>$ tag.

6. Continue to work with the IOOS/Google working group to explore the use of KML as a data format for transferring ocean observing data.

7. Build a Google Maps web interface for Massachusetts that integrates

a. Search capabilities (provided by Google)

b. GeoServer web services and their GIS services

c. SOS services

d. OPeNDAP/Thredds

e. KML

f. ERDDAP 
8. Improve web-based interfaces to access and visualize data from OPeNDAP, Thredds, and ERDDAP, WFS, SOS, and WCS. This will allow data links to be embedded in the <NetworkLink> section of the wrapper KML and will allow users to directly connect to that data with web tools. These tools should include thin client access to Thredds/Opendap and SOS services with data visualization and analysis tools.

9. Support the finalization of the proposed unstructured grid standard, and develop a visualization and analysis tool based on the unstructured grid convention would move the standards process forward and open up access to a range of currently non-standard data via OPeNDAP and THREDDS.

10. Continue to support the implementation of the OGC standards, KML, WMS, WFS, and WCS.

11. The use of middleware, such as ERDDAP, to manage transformations of scientific data for wider consumption should be implemented more broadly.

12. Use of ncWMS and the other OPeNDAP/THREDDS map services technology to expose gridded model and satellite data via OGC services.

13. Build a "datapedia" which will allow users and providers to share information on data and reports.

14. Evaluate possible implementation of the ESRI GIS Portal Toolkit as a portal for regional data in combination with MassGIS GeoServer.

15. Work with the user groups to determine priorities on the data needs and coordinate with the data providers to make that data available in the required formats and with tools that assist in ocean management decision making. We suggest the following :

- Hold user group/stakeholder workshops to determine priorities on the data needs and coordinate with the data providers to access the data in standard formats.

- Work with the user groups to promote cross-disciplinary coordination of data through indicator datasets and sensitivity indices.

- Work with the user groups to develop tools for decision support, e.g user-specified weighting of various themes rules-based algorithms for screening-level analysis.

16. Coordinate with NERACOOS, MARCOOS and GOMODP, and leverage existing projects in the region that already serve priority data (i.e. OBIS, TNC Ecoregional assessment, NOAA marine cadastre)

17. Develop and continually update a phased implementation plan that is based on user priorities, and for each task, clearly identifies potential partners, existing efforts that can be leveraged, potential funding opportunities, and long term maintenance/responsibilities.

\section{- 44 - INTERIM DRAFT}




\section{Data Management Network Schematic}

\section{Data Providers}
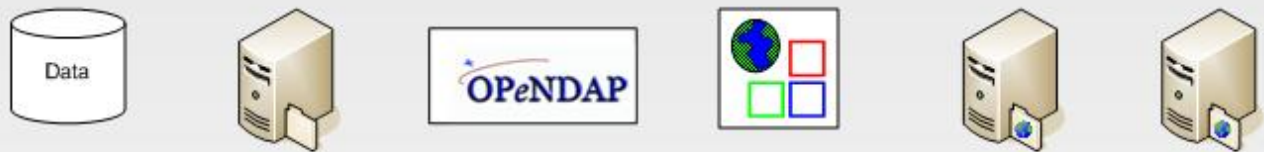

Databases

GIS Servers

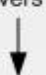

OPENDAP

Web Services

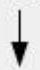

FTP/File Servers

etc.....

Data sources furnish KML files representing metadata existing search capability to interrogate available data catalog, available output formats and options
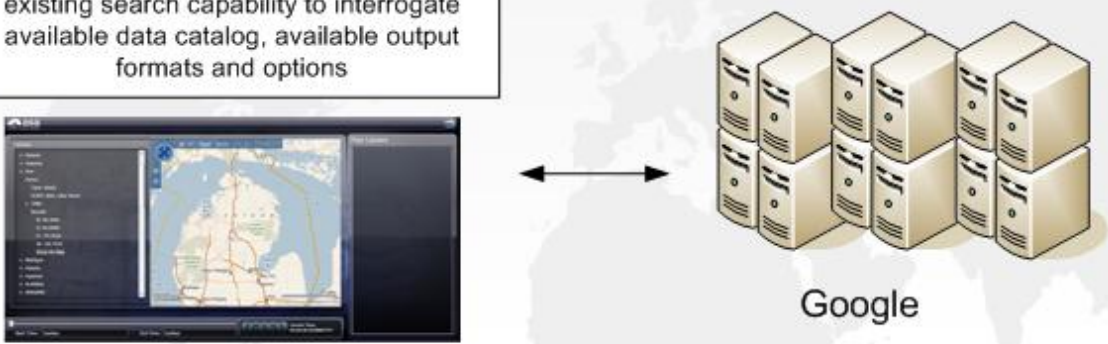

Google

Google Bot Indexes KML files for search

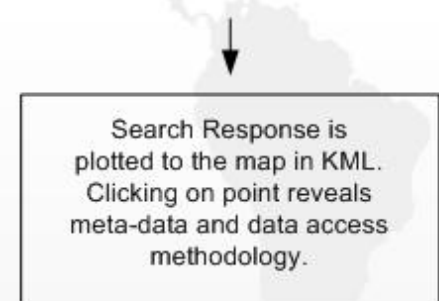

Interrogation of data allows plotting, ingest, download, etc. depending on the data type that is interrogated.

Application determines access method based on data type and metadata. Data network provides access to various types of data, GIS, file data, database data, tabular data, graph data, informational data, etc. Network offers end user tools to work with data that are available.

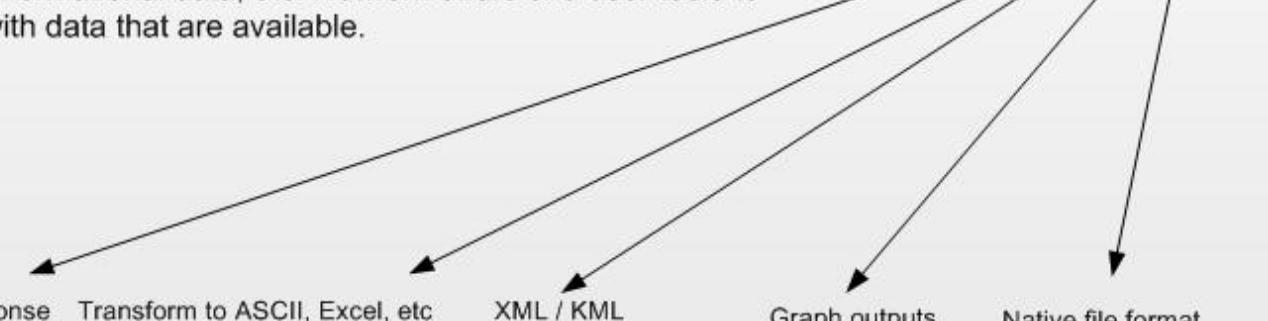

Image Response Transform to ASCII, Excel, etc

$\mathrm{XML} / \mathrm{KML}$ Graph outputs

Native file format
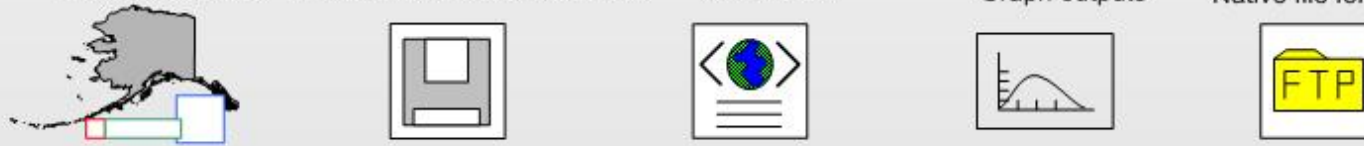

\section{Data Network Conceptual Design}




\section{Conclusions}

ASA worked with the Massachusetts Ocean Partnership (MOP) to write a conceptual design report for an ocean data network to support ocean management planning in Massachusetts. An ocean data network is an infrastructure of data, systems, services, and tools that allow a variety of users including the public, coastal managers, and research scientists to access "live" and archived data related to coastal and ocean management. This may include maps, observations, model data, and non geographic data such as reports and images, and the data may come from a wide variety of "data providers". The report reviewed existing observation systems, regional initiatives, existing infrastructure, data management concepts, and user issues. We recognized that a considerable amount of work is on-going related to the integration of disparate data and interoperability standards and we emphasized the need to leverage existing efforts, including the existing GIS infrastructure at MassGIS.

We suggested that 4 concepts were the key to a successful data network design:

- Focus on interoperability and the use of existing science tools connected to commercial Web 2.0 concepts

- Leverage existing global, national, and regional efforts and use the general guidelines provided by the National IOOS DMAC concept of operations

- Provide tools and "glue" to integrate legacy systems as opposed to redesigning components

- Focus on meeting user's needs

We recognized that there are a number of challenges, some related to technology ("glue") needed to connect disparate data streams to different user groups, and some related to user issues and the need to define user needs through a series of "use cases" so tools can be applied to the data for decision making.

We feel strongly that the ability for the data network to bridge the gap between the science and GIS world with the rapidly evolving Web 2.0 world is key to effectively finding data and accessing data with existing commercial tools, and that this concept can potentially lead to significant breakthroughs. 


\section{Acknowledgements}

Thanks to the folks who provided us input during preparation of this report:

Steve Ansari - NOAA NCDC

Julie Bosch - NOAA NCDDC

Katie Budreski - Stone Environmental

Rob Cermack - Alaska Ocean Observing System

Peter Cornillon - URI, OPeNDAP

Jeremy Cothran - Southeast Coastal Ocean Observing System

Aleda Freeman - MassGIS

Peter Giencke - Google Inc.

David Healy-Stone Environmental

Dan Holloway - URI, OPeNDAP

Christian Jacqz- MassGIS

Alan Leonardi - NOAA liaison to Google

Daniel Martin - NOAA CSC

Roy Mendelsohn -NOAA NMFS

Sharon Mesick - NOAA NCDDC

Rost Parsons - NOAA NCDDC

Dan Sampson - MassCZM

Rich Signell - USGS

\section{References}

Boeing Company, Joint Navy/NOAA NESIDIS IOOS Demonstration Project, System Design Specification Report, CDRAL A003 (Draft), October 14, 2004.

Charter of the Interagency Working Group on Ocean Observations, issued by the Joint Subcommittee on Ocean Science and Technology, Committee on Environment and Natural Resources, National Science and Technology Council, December 2006. Executive Summary.

Committee on Archiving and Accessing Environmental and Geospatial Data at NOAA, National Research Council, Environmental Data Management at NOAA: Archiving, Stewardship, and Access, 2007.

\section{- 47 - INTERIM DRAFT}


Dyke, Stamp, Chamberlain and Ullyett. GIS and Policy Making: Beyond Aesthetically Pleasing Pictures. Scientific Officers Environmental Information Centre Institute of Terrestrial Ecology. 1996. 30 Jan 2009.

Gulf of Maine Ocean Data Partnership, Technical Guidance for Data Sharing Version 1.2, November 2007

Hanke, John and Jack Dangermond,"State of the Geoweb", Where 2.0 Conference, 2008

International Oceanographic Data and Information Exchange (IODE), Intergovernmental Oceanographic Commission of UNESCO. Marine Data Management: we can do more, but can we do better? Jan. 2007. 30 Jan. 2009.

Joint Oceanographic Institutions, ORION Cyberinfrastructure: Concept of Operations, Version Or8, November 11, 2005.

Lockheed Martin, Integrated Ocean Observing System (IOOS): Conceptual Design, 2006

Long, Lauren, Geno Olmi, and Dave Easter, "Integrated Ocean Observing System Regional Association Boundaries and Federal Agency Relationships" NOAA Coastal Services Center, December 2007. National Office for Integrated and Sustained Ocean Observations, Data Management and Communications Plan for Research and Operational Integrated Ocean Observing Systems, Ocean.US Publication 6, March 2005.

National Office for Integrated and Sustained Ocean Observations, The First U.S. Integrated Ocean Observing System (IOOS) Development Plan, Ocean.US Publication 9, January 2006.

National Office for Integrated and Sustained Ocean Observations, The United States of America's Contributions to the Global Ocean Observing System 2007, Ocean.US Publication X, May 2007.

National Office for Integrated and Sustained Ocean Observations, IOOS Development Plan Addendum, Oceans US Publication No. 9-A1, Washington DC, 2007.

NOAA, IOOS Program Office, Data Integration Framework (DIF) Functional Requirements Document, Version 1.0, November 19, 2007.

NOAA, IOOS Program Office, Data Integration Framework (DIF) Master

Project Plan, Version 1.0, November 8, 2007.

NOAA, IOOS Program Office, Data Integration Framework Concept of Operations, Draft Version 2.0, December 3, 2007.

NOAA, IOOS Program Office, Data Management and Communications Concept of Operations, October 2008.

Raytheon Corporation, U.S. Integrated Ocean Observing System, Volume 1-IOOS Conceptual Design, August 31, 2006.

U.S. Commission on Ocean Policy, An Ocean Blueprint for the 21st Century, Final Report, July 2004.

\section{- 48 - INTERIM DRAFT}




\section{Websites}

Joint Oceanographic Institution, http://www.joiscience.org/ocean_observing

National Data Buoy Center, http://www.ndbc.noaa.gov

NOAA IOOS Program, http://ioos.noaa.gov

Ocean Research Interactive Observatory Networks (ORION) Program, http://orionprogram.org/OOI/default.html

NOAA Science Advisory Board Working Groups,

http://www.sab.noaa.gov/Working_Groups/standing/index.html

Federal Geographic Data committee, http://www.fgdc.gov/metadata

GeoServer, http://geoserver.org/display/GEOS/Welcome

Massachusetts Ocean Resource Information System (MORIS) CZM's Online Mapping Tool, http://www.mass.gov/czm/mapping/index.htm

Massachusetts Geographic Information System, http://www.mass.gov/mgis/massgis.htm

Integrated Ocean Observing System (IOOS), http://ioos.noaa.gov/

Ocean Research Interactive Observatory Networks, http://www.orionprogram.org/OOI/default.html

\section{Reports \& Meetings}

Boeri, R., Haney, R., Knisel, J., Mooney, K., Orfant, J., Sampson, D., Sprague, J., Wilbur, A., Barnhardt, W., Fields, L., Hanson, W., Jaedtke, W., Kennelly, J., Liebman, M., Murray, R., Ramsey, J. 11-21-2008. Regional Sediment Resource Management Workgroup Report.

Carlisle, B., Buchsbaum, R., Callaghan, T., Chambliss, E., Colarusso, P., Ford, K., French, T., Mayo, C., Killerlain-Morrison, K., Sampson, D., Sprague, J., Szczebak, D., Tyrrell, M., Wilbur, T. 11-26-2008. Habitat Work Group Report.

Diodati, P., Dean, M., Armstrong, M., Correia, S., Ford, K., Griffin, M., Glenn, B., Hickey, M., Hoopes, T., King, J. Fisheries WorkgroupFinal Report.

Ducsik, D., Lloyd, N., Mastone, V., Rowcroft, J., Stella, A., Mahlstedt, T., Bell, E., Ryan, R., Agganis, G., Carr, A., Roach, L. 11-21-2008. Report of The Workgroup On Ocean Recreational And Cultural Services.

EOEEA, DOER, and CZM Renewable energy working group members. 12-03-2008 Draft Report of The Renewable Energy Working Group.

Ocean Science Advisory Council Meeting Summary. December 8, 2008. Boston.

Ocean Science Advisory Council Meeting Summary. January 14, 2009. Boston.

\section{- 49 - INTERIM DRAFT}


Stone-Environmental. 12-18-2008. DatasetListing_LongReport.

Stone-Environmental. 12-18-2008. DatasetListing_ShortReport.

Stone-Environmental. 12-18-2008. DataStewardListing_Report.

Stone-Environmental. 12-18-2008. Master MOP Data Inventory

Washburn, B., MacGaffey, N., Wellock, B., Lynch, B., King, M., Wingate, M., Gelzer, C., Farmer, G., Glavin, G., O'Donnell, E. 11-21-2008. Draft Report Of The Transportation, Navigation, Infrastructure Working Group.

Jan Seys (Flanders Marine Institute (VLIZ)), Jan Mees(, Ward Vanden Berghe (Flanders Marine Data and Information Centre), Peter Pissierssens (Intergovernmental Oceanographic Commission of UNESCO), 2007.

Lockheed Martin Transportation and Security Solutions

OGC ${ }^{\circledR}$ KML Standard Development Best Practices, OGC ${ }^{\circledR}$ 08-125r1, 2009-02-04

ESRI ${ }^{\circledast}$ Geospatial Portal Technology, ESRI White Paper, June 2007

Creating and Maintaining a GIS Portal - Management Considerations, July 2008

GUIDE FOR IOOS DATA PROVIDERS, VERSION 1.0, June 2, 2006, Ocean.US IOOS DMAC Steering Team Northeast Regional Association of Coastal Ocean Observing Systems Progress Report, June 30, 2008

M. L. Spaulding, A. Grilli, and S. Grilli, URI Ocean Engineering, Technology and Energy Resource Based Screening Analysis

\section{Acronym Appendix}

CZM - Massachusetts Office of Coastal Zone Management

DIF - Data Interchange Format

DMAC - Data Management and Communications

EOEEA - Office of Energy and Environmental Affairs

EPRI- Electric Power Research Institute

FGDC - Federal Geographic Data Committee

GCMD - Global Change Master Directory

GEOSS - Global Earth Observation System of Systems

GIS - Geographic Information System 
GOOS - Global Ocean Observing System

GOS - Geospatial One Stop

IEOS - U.S. Integrated Earth Observation System

IOOS - Integrated Ocean Observing System

$\mathrm{KML}$ - Keyhole Markup Language

MassGIS - Massachusetts Geographic Information System

MOP - Massachusetts Ocean Partnership

MORIS - Massachusetts Ocean Resource Information System

MRIP- Marine Recreational Information Program

NASA - National Aeronautics and Space Administration

NECOFS- Northeast Coastal Ocean Forecast System

NERACOOS - Northeastern Regional Association of Coastal Ocean Observing Systems

NMFS- National Marine Fisheries Service

NOAA - National Oceanic and Atmospheric Administration

OGC - Open Geospatial Consortium

OOI - Ocean Observing Initiative

OPeNDAP - Open-source Project for a Network Data Access Protocol

SOA - Service Oriented Architecture

THREDDS - Thematic Realtime Environmental Distributed Data Services

SDE - Spatial Database Engine

USGS- United States Geological Survey

$\mathrm{XML}$ - eXtensible Mark-up Language 